\section{BRUTALISMOS EDUCATIVOS. LA ARQUITECTURA COMO NUEVA} PSICOGEOGRAFÍA SOCIAL

EDUCATING BRUTALISMS. ARCHITECTURE AS NEW SOCIAL GEOGRAPHY

Patricia de Diego Ruiz

\section{INTRODUCCION}

a arquitectura difícilmente puede obviar la in- lluencia que, de manera más o menos deterel modo de vida de sus habitantes. La manera en la que un edificio se implanta en un lugar, asi como las características propias que lo definen a nivel fisico y material, condicionan al hombre en el desarrollo de sus funciones y en el modo de comprensión especifico del entorno. Esta capacidad inmanente la arquitectura adquiere una mayor dimensión cuando se tratan edificios escolares y colegios. La responsabilidad se acrecienta al manejar personalidades incipientes y capacidades críticas en formación

ados del siglo pasado, en las décadas posteriores a la finalización de la segunda Guerra Mundal, consthy un momento intereante de estudio para vislumbrar las interacciones aue el enfogue educalivo y pollicopurde tener en su traslacion erqulectónica. La primacía establecida de unos objellvos sobre otros y el orden de jerarquia on la toma de decisiones condicionó de manera determinante el planteamiento del

proyecto arquitectónico asi como los recursos manejados por los arquitectos en el diseno de la arquitectura escolar y su alcance.

El caso más celebre y estudiado de este contexto geos Colegios prefabricados de Hertordshire. Ideados por David Medd Junto a empresa Hils, constituyeron desde el inicio un ejemplo

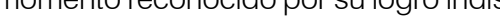
oulible de provisión de doscientos colegios en un periodo de quince años y con un coste ajustado. Pero los colegios de Hertordshire lograron introducir un cambio de dimensión ontológico de mucho mayor alcance en el debate britanico que no resulta tan conocido. Nicholas Bullock señala como John Summerson identficaba la edificación escolar como una tendencia en busca de principios (...) principios anunciados como edificios"1 y J.M. Richards sostenía que definilan "el rostro de una nueva y especialmente inglesa arquilectura". Fueron el despegue de un nuevo enfoque arquitectónico propiciado desde la esfera politica y pública en el que ingenieros y constructores trabajaban de modo colaborativo con los arquitectos para el planteamiento de sistemas constructivos capaces de 1. BULLOCK, Nicholas. "Reconstruction, School Bulding and the Avant-Garde". Contribución al Congreso "Team 10-between Modernity and the Everyday, 2. Ídem. 
ser implantados a gran escala como pre-soluciones. La particularidad de su concepción como un mecano completo en malla basado en el módulo de ocho pies por tres
pulgadas, permitió prefabricar no sólo aulas, sino el total del colegio, e introdujo un considerable grado de flexibiIdad que permitía implantaciones especificas y numerosas combinaciones en soluciones particulares (figura 1). satisfaciendo las domandas del momento de pedagogos .

Los Colegios de Herftordshire fueron admirados inicialmente tanto por la angry young generation como por los arquitectos más vanguardistas de la generación antehritánica que lideráí brlánica que lics coria sintácticos de este nuevo enfos valoes pragnálicos y ha de supericiales ideates estísticos, con percibido como un camino restrictivo por este grupo de prquitectos. Su planteamiento se consideraba inu para poder adecuarse a las demandas sociales reales complejas, y hasta perverso, por perpetuarse en ellos la rapidaz y la economí como propósitos prioritarios a los que quedaba subordinada la forma arquitectónica La tipificación se impoń al pensamiento de la mejor solución arquitectónica concreta

principios certeramente detectada por Summerson y que fue tan crucial para la nueva generación de arquitectos, la industrilización pasó de ser la respuesta única, a ser un factor importante que se sumí naturamente. De la solución generalizada como sistema se pasó a la prefabricación personalizada y a la

Introducción de técnicas tradicionales de bajo coste para poder competir en términos económicos. El foco de aten-

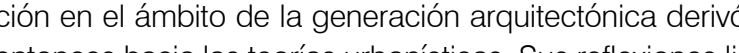
(ition deraban la apertura de caminos novedosos que trataban de comprender el entorno y rediseñarlo en terminos conemporáneos, en detrimento de un C.I.A.M. decepcionanmientras fonana suén en lad propositua y visionaria entre for entre forma y el total de las necesidades humanas y de sus respuestas

Las propuestas psicogeográficas eran bien conocidas en el círculo de vanguardia inglés, tanto en su desarno europeo a través de los situacionistas franceses lynch. La sensiblidad brutista 5 conurá con Kevin Lort. La senibi de movilided al percibir su aumento cono característo actuat de la era mecarizada y propia de una sociedad en perpetuo cambio Se centráá en el potencial del tránsito peaton l lo asimilá cono instumento de captacín y conocimiento el entón como pero uilizá á cambén como un mecanismo de transformacín person lat secuencial paulatino de la realidad arquitectónica cotidiena:

Para ello se inspiran en la 'dérive' descita en la inpacional Situacionista como "un modo de comportamiento experimental ligado a la condición de sociedad urbana: una técrica de pasaje en tránsito a tracísad ambientes variados"6. correspondín a partes de la ciudad que emanaban

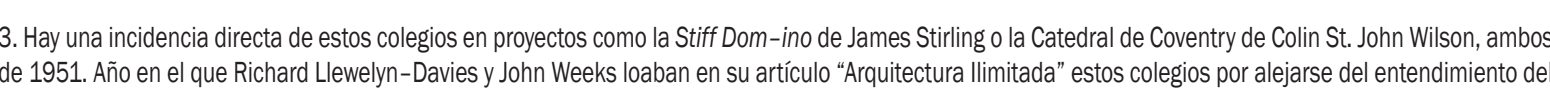
edificio como objeto completo perfectamente definido y cerrado.

4.Maxwell Fry señalaba como estos colegios prefabbricados resuttaron monótonos y básicos, planteados según una línea puramente racional que sólo satisfarla a Walter Gropius, y alentaba una alternativa que discurriera "entre una industrialización rigurosa y l la severa dirección estatall", donde "la energía creativa maltrecho oue pronto tuvieron estos colegios y se cuestionaba si las técnicas lijerers y los materiales utilizados no eran demasiado transitorios para unos edificios relativamente estables y permanentes. 5. La aplicación de los arquitectos de estas teorías se apartará de la interprotación de Reyner Banham que aplaudirá la "téćnica de emociones y compor-

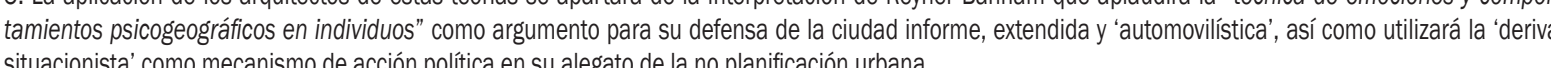
'tuacionista' 'como mecanismo de accion politica en su alegato de la no planificacion urbana. 5. KNABB, Ken. Preliminary rroblems in Constructing a Situation, p.45. En: Ken KNABB (ed): Situationist Intermational Anthology, Berkley: Bureau of Public
Secrets, 1995, pp. 43-45.
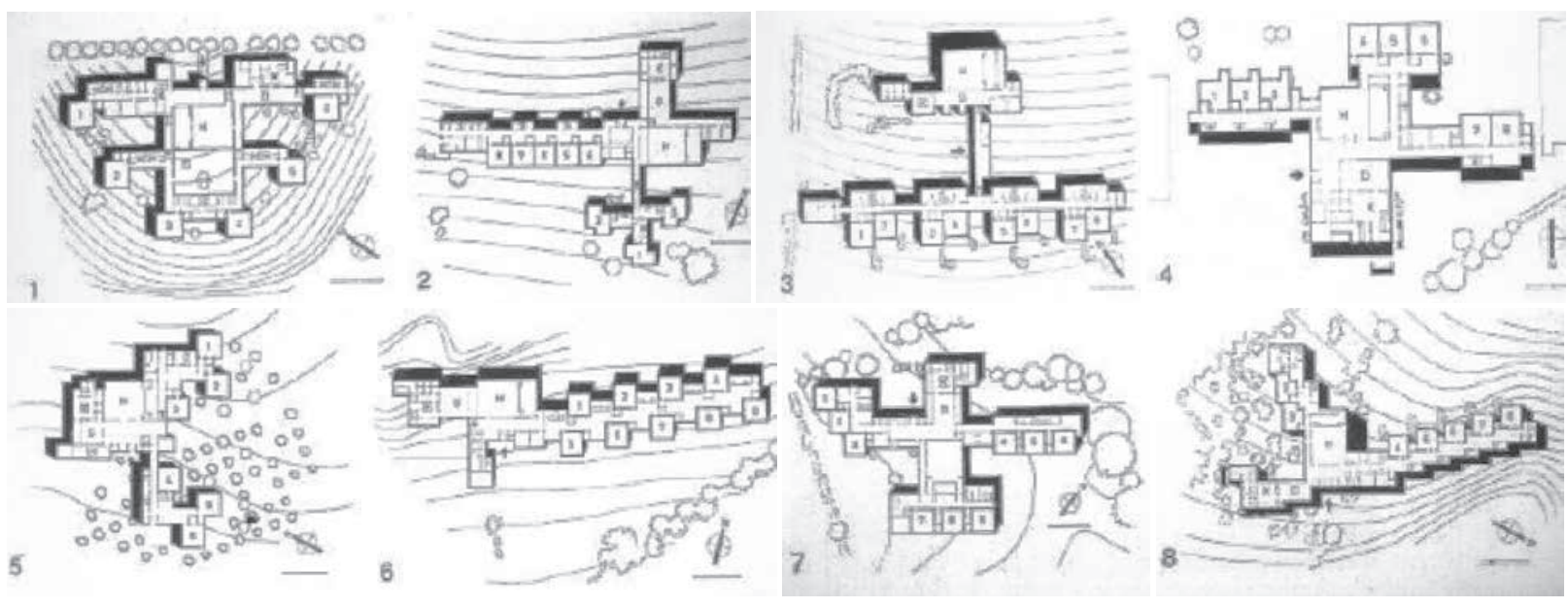

desarrollo de un sentimiento o ánimo asociado a su caácter y efecto; pero también eran referidos a la propia condición geográlica del lugar como pequeño barrio o de los situracionistas con Lynch no sólo respecto a su de los such du interés común en las 'unités d'ambinne' yen los es. Notas sobre la Satisfacción urbana' de 1953, declara'Da estar "Nosotros estamos aqu' conciencialos con los efectos psicológicos y sensuales de a forma física do ciudad"s A través de sus trabajos posteriores y sus colaboraciones con Gyorgy Kepes ahondará en el estudio de los elementos más determinantes en la percepción del ambiente urbano, haciendo un registro exhaustivo de secciones de calles, pasajes $y$ todo tipo de salientes or namentales y distintivos como rejas, balcones, bow-windows o rellanos de entrada. Esta atención a la rugosidad y protuberancias de las fachadas tendrá un parosidad en el uso de salientes, pliegues y compleiidad otorgada la envolvente arquitectónice en los colegios brutalistas que son usados como signos que pautan, amenizan y clarifican el ámbito doméstico cotidiano. Su alejamiento del acabado material abstracto y su rechazo al uso del muro cortina como indiferente de la actividad interior son manifestaciones de esa voluntad de hacer legible la arquitectura por medio de un aspecto perceptible que derva de la articulación de diversas formas particularizadas.

surgidas del estudio previo minucioso del crisol de necesidades interiores y la potenciación de sus cualidades distintivas. En este sentido, este planteamiento es deudor del trabajo de Lynch, cuyo esfuerzo por comprender as imágenes mentales concebidas por los habitantes de as ciudades americanas se sustentaba en el estudio de valor característico de la forma visual ${ }^{9}$.

a palabra 'imagen' fue rescatada del ámbito urbanístico y puesta en relación con la arquitectura en su artículo sobre el Nuevo Brutalismo. Su estipulación de termino como una de be tres caracteristicas propies de a arquitectura de este movimiento ha dado lugar a la interpretación generalizada de su traslación más direccomo cualidad escultórica y visualmente impactante Sin embargo, Laurent Stadler ha destacado la condición operativa del uso del concepto desvelando varias de las ramificaciones que un término tan complejo y polisémico como éste conllevaba en el debate de los años cincuenta ${ }^{10}$. El análisis de Banham en su aplicación a la arquitectura aclaraba que "el edificio debe ser una entidad visuinmediatamente aprehensible; y que la forma captada por el ojo debe ser confirmada por la experiencia del edificio en uso"11. Es esa naturaleza de la imagen como configu ración que trasciende lo visual afectando a las emociones, y a través de la experiencia permite la asimilación de la forma arquitectónica hasta convertirla en imagen mental interiorizada que enlaza con el concepto de entorno vivencial que estudia y reclama la Psicogeografía.

Jnch Debord. About two Psychogeoograhiess'. En: Cartographica, v.45, 3, pp.185-200.

8. Íbid, p.190

9. Trabajo que culminó en 1960 con el libro La Imagén de la Ciudad, donde Lynnch defiende las unidades elementales de comprensión constituidas como imà genes 'altamente identificables', en una critica a los espacios informes, anodinos y monótonos que define como generadores de confusión. 10. STADLER, Laurent. " "New Brutalism','Topology' and "Image'. 'Some remarks on the architectural debates in England around 1950". En The Journal of Arch

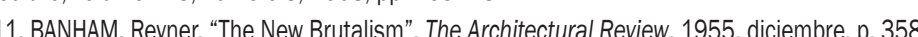




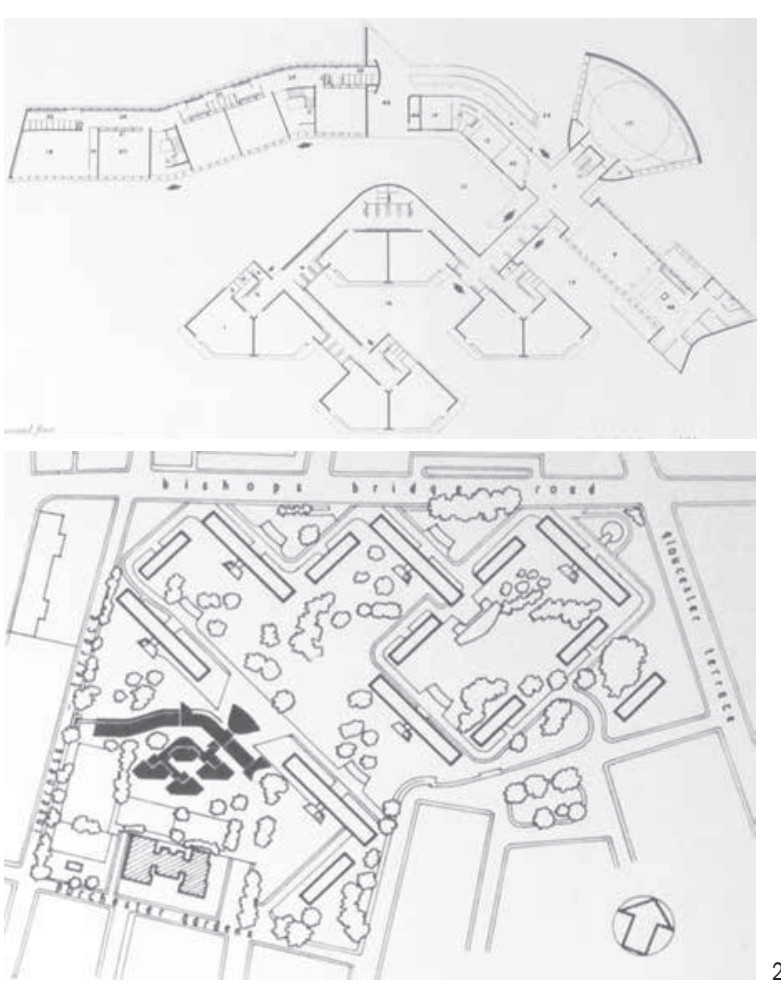

2. Plano de situación del conjunto residencial de Bishop Bridge y planta baja del conjunto del Colegio de

3.Cluster' de aulas infantiles y algunas posibles
disposiciones mostrando tanto la variabilidad de agrupación como la capacidad de alteración interna 4. Particas porizaín de cada activids su mobilario. tintos volúmenes, posicín y cualidad de cerramientos. El edificio de primaria oculta las áreas recreativas de la calle y su sinuosidad evita la monotonia perceptiva de los pilares prefabricados aplantillados. Las mayores piezas de comedory auditorio multusos se disponen cerca dé la entrada para posible
segregación mientras el área infantil se despliega en un ámbito interior más tranquilo y apartado. 5. Area infantil de aulas orientadas a sur con espacio exterior asociado y alfézar como mobiliario integrado que alientan una educación no-institucionalizada. alturas y aperturas según actividades y proyección al entorno.

EDUCANDO NIÑOS

El colegio de Primaria Hallield en Paddington

Las principales características relativas a la educación primaria vigentes a mediados del siglo pasado en Gran Bretaña quedaron perfiladas en el Boletín no1 que el Ministerio de Educación publicó en el año 1949. La distensión parcial de esta disposición en materia educativa permitió que, aunque el Colegio Hallfield que Denys Lasdun y Lindsey Drake proyectaron para el distrito de Paddington se adhería inexorablemente al contenido de citado boletín, el resultado arquitectónico estuviese inusualmente alejado de su arquetipo tradicional.

El Colegio de Hallfield comenzó a ser diseñado en vo Brutalismo'i2 su radicalismo amable supuso la pieda

angular que proclama el derecho a la búsqueda de una orma propia cor aradigma que rompe tanto con la ortodoxia 'moderna' británica como con la apuesta pública de arquitectura escolar industrializada ${ }^{13}$. Aquella ordenación flexible y abierta admirada en los Colegios de Hertfordshire que conjugaba el edificio como un sistema de partes moduladas, sufre una transformación seminal para plantear la disposición arquitectónica como una estructura relaciones, como un organismo heteromórfico (figura 2) y variado ${ }^{14} \mathrm{El}$ caleidoscopio de formas desplegadas para el proyecto no qued reñido con la atención a la seriación propia de la segunda era industrial, pues dispone el área infantil como una agrupación en racimo o 'cluster'15 donde todas las células tienen el mismo volumen, coincidente con el total del aula

12. Su diseño comienza previamente a la aparición del término 'Nuevo Brutalismo' que introdujo Alison Smithson en su artículo “Soho House" de 1953. Por esta razón no suele relacionarse con el, aunque Hunstanton presenta un destase temporal similar, y por un manejo de los materiales todavía deudor de la

3. El trabajo de Lasdun y Drake con la firma Tecton en el barrio, que sigue presupuestos higienistas del Movimiento Moderno, no les afectó para articular una (4)

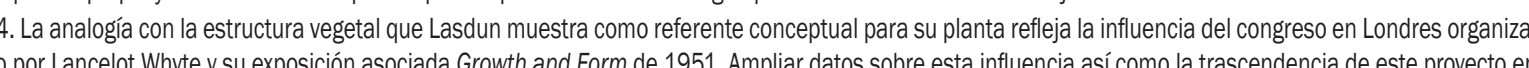
a trayectoria de Lasdun en, DE DIEGO, Patricicia."Denys Lasdun y el proyecto para la Universisidad de East Anglia. Síntesis de una filiación orgánica"." Cuaderno de Proyectos de Arquitectura, n.8, 2015, pp. 99-73.

15. El termino cluster era usado por Kevin Lynch y fue popularizado a través de su articulo en la revista Scientific American en el año 1954. Denys Lasdun

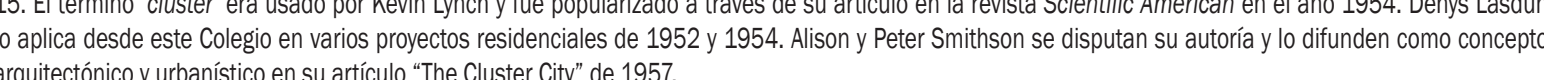
$\Delta$

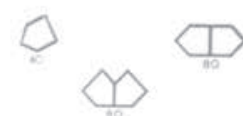
$(\mathrm{D})$
$\Delta$

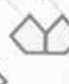

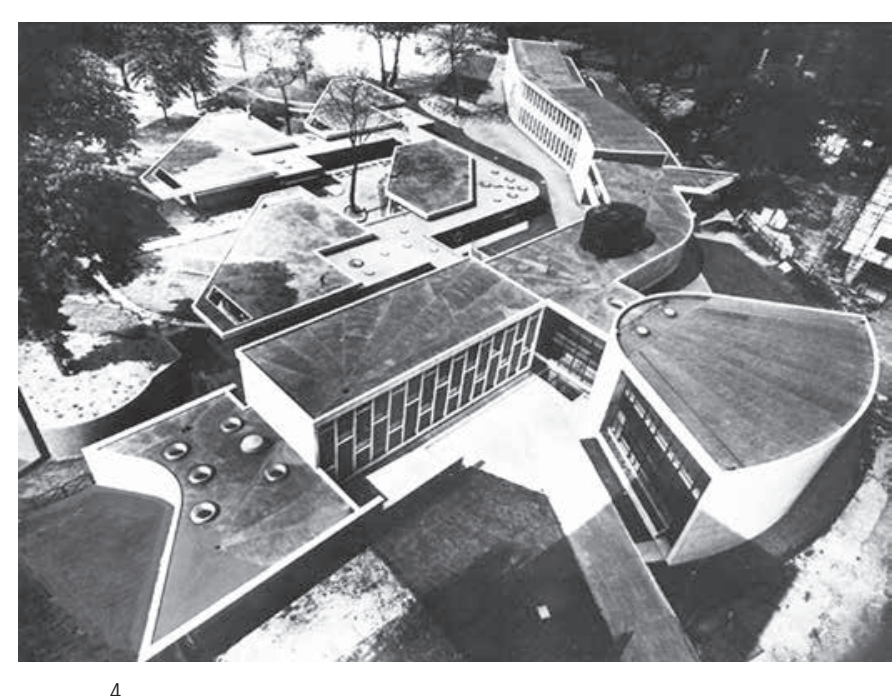

mecánica existente en los colegios industrializados. Pero esa repetición de la forma no surge de imposiciones técnicas constructivas o de un sistema previo. Lasdun y Drake plantean una forma espe cifica pentagonal que no sôlo permite la agrupación variada generando unidades superiores diversas, sino que deriva de la búsqueda de la mejor forma capaz que pueda albergar múliples conilguraciones internas para atender a las necesidades de juegoy actividades cambiantes que son convenientes para la estimulación de este grupo de edad (figura 3)

Lastructivas y materiales se combinan

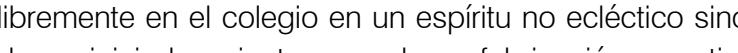
despejuclado, mientras que la prefabricacion, que tlee nugr deslacádo en su construcción, no se aplica uso se amolda a las carterísticas funcionates de card

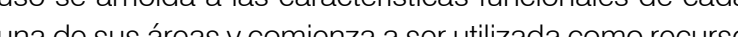
expresivo a servicio de la tome dominente para do un carácter distintivo a cada parte.

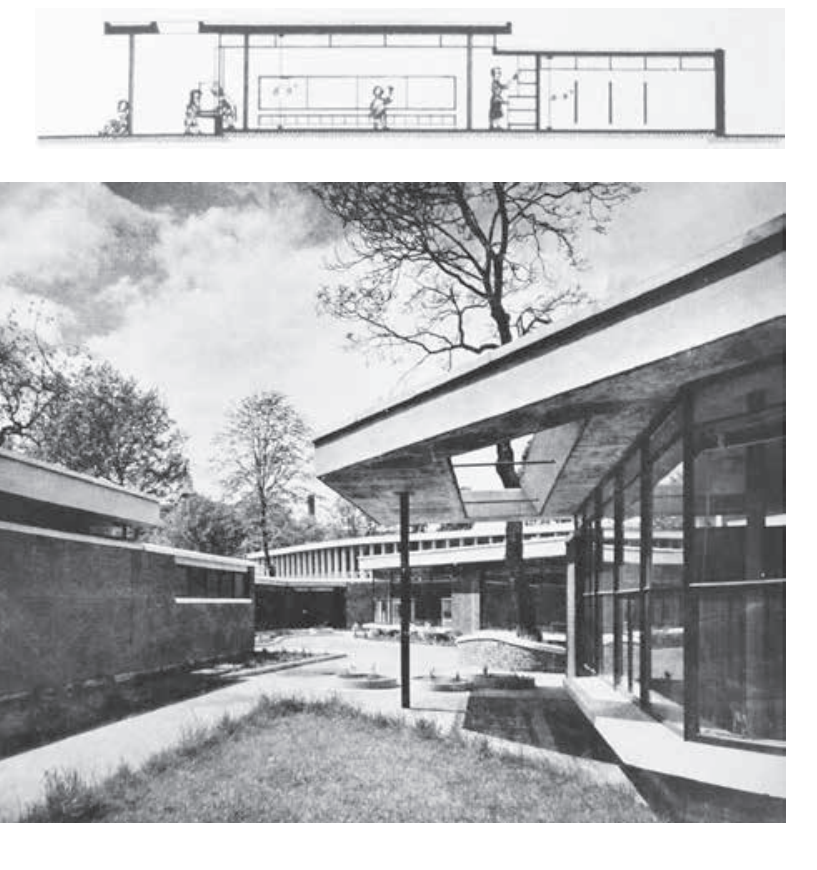

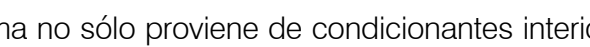
res sino que es sensible igualmente a las fuerzas extehores signilicadas, en el caso de esta arquitectura, en la atención a las preexistencias. El Colegio Hallield asume resides comu pes a la disposición general de los bloques verger en entracos que se inserta para hacerlos con-

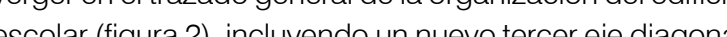

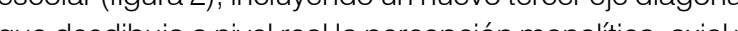
egular que pudiera tenerse del centro.

La aluraconside ble de los bloques iesidenciles que La elegio generando un gredente de privacidad progresivo descle la calle trasera de llegada hacio el jardin al que se abre (figura 4) Volúmenes gecrientes y una alta sensibitdad en el trabajo de paramentos y aperturas logran espect icos niveles de intimidad y unas áreas de juego infantil que

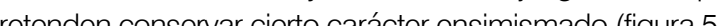
Todo el despliegue de particularización que presenta este colegio hacia la diversidad de usos y necesidades 
6. Vista de una de las entradas que evidencia la

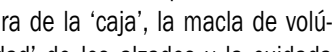
menes, la 'rugosidad' de los alzados y la cuidada
elaboración del diffrute asociado a las circulaciones. 7. Axonométrica del colegio Wokingham que muestra una forma distintiva estrelladada para áreas comunes y el encadenamiento sucesivo de la 'unidad tipo':
aula, espacio de aseo y almacenaje, zona exterior aula, espacio de aseo y almacenaje, zona exterior
propia delimitada. 8. El uso de las superficies vidriadas inclinadas en
cubierta desdibujal la sensación de cerramiento y ge. nera un lugar semiexterior climatizado pero volcado

a la naturaleza, la climatología yel contexto.

que requiere cada edad de los niños que lo ocupan se hace posible, en gran parte, al uso de la circulación como

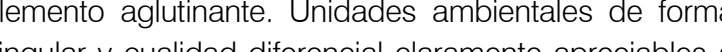
nivel perceptivo ligadas por un magma a recorrer Los espacios de tránsito pasan de ser elementos residuales neludibles que dan acceso a los varios habitáculos, a ser espacios activos cruciales que añaden un valor propio que mejora sustancialmente al edificio.

Las diversas anchuras de los espacios de circulación esultantes de la integración volumétrica de estancias de aseo, almacenaje, superficies de descanso y elementos de comunicación, junto a la manera espećfica en la que en cada uno de los espacios de tránsito se relaciona con la mirada hacia el interior y el exterior, hace que la red de pasillos generada para el colegio se convierta en una pulsación espacial y fenomenológica dinámica y fluida. La multiplicación de conexiones con el entorno inmediato a diferentes cotas y el desdoblamiento de accesos induce a los niños a variar surecorrido hasta el aula con la posibilidad de al menos dos opciones distintas (figura 6 ).

La comprensión del Colegio de Primaria de Hallfield no se produce de manera inmediata y requiere necesariamente del recorrido para su entendimiento. Progresivamente se desvela en secuencias que intercalan espacios dinámicos y puntos focales estáticos que refuerzan la generación de 'ambiances' que producen imágenes mentaes de reconocimiento y asimilación del espacio. Los leves giros en la geometria de la planta y los cambios en la opacidad del cerramiento materializan la vocación clara por parte de los arquitectos de crear un ambiente lúdico y sorpresivo que incite a realizar itinerarios y exploraciones libres dentro y fuera del edificio. Son circulaciones elongadas pero de cualidad diversa que construyen entornos de aprendizaje y de estimulación de la movilidad.

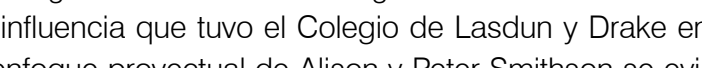
列 ceptivamente compacta y regular presente en su famosa Escuela de Hunstanton, a una fragmentada y flexible con a que diseñaron en 1958 el Colegio de Wokingham. E parámetro de la movilidad como dinámica generativa de a disposición arquitectónica se mantiene a través del uso de la circulación como principio estructurador alrededor del cual se van enlazando piezas diferenciales y singulares. Un paquete programático de aula y espacios asociados a modo de pequeña subcomunidad se repite seriada y rítmicamente a lo largo de una directriz no disciplinada que rememora una espiral de crecimiento (figura 7). Este proyecto es, según Kenneth Frampton, "la aplicación más elegante del principio del 'cluster' socio-cultural de los Smithson"16 que desarrolla una 'ética' propia que, para e crítico, sólo vuelven a aplicar con similar maestría en su casa de fin de semana de Upper Lawn.

El uso de las "formas abiertas que son 'creadoras-de ugares"17 acompaña el cambio de paradigma permitiendo que el colegio de Wokingham, a través de su geometría no euclidiana y por medio de su implantación, envuelva y moldee el espacio creando la sensación de una concatenación de recintos. Esa atención al espacio acotado es reclamado como necesario en la percepción y desarrollo nfantil tambien por los Smithson. En su aplicacion en los exteriores a través de la dellimitación de caminos y muros, el Colegio de Wokingham pretende rescatar la escala doméstica del espacio público representada en el collage que realizaron con lasfotografilas de Nigel Henderson sobre el juego de los niños en el barrio de Benthnal Green ${ }^{18}$. Las aulas se onchan a sury pueden hacer uso de esas reas externas como espacio educativo no reglado al aire

16. FRAMPTON, Kenneth: Souvenirs du sous-dévelopment. LA'Architecture d'Aujourd'hui, 344, janv-fev, 2003, p. 94.

8. Apropiación espontanea y lúdica de calles y espacios urbanos cotidianos reflejada en las fotografías del barrio Iondinense que Alison y Peter Smithso

.
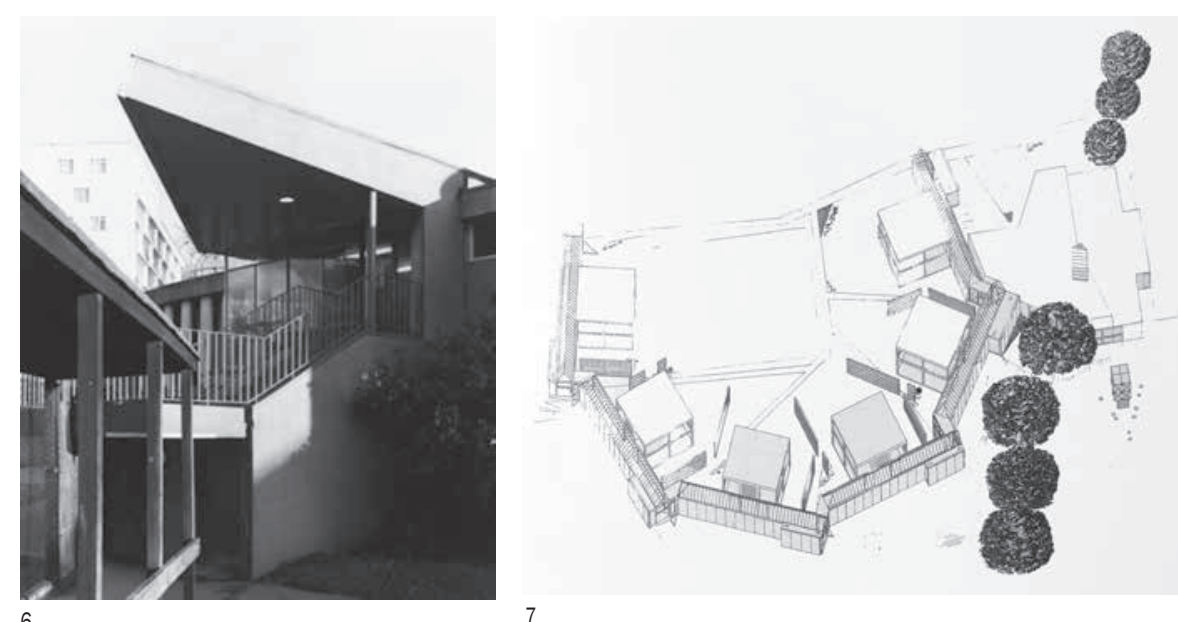

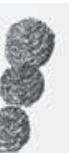

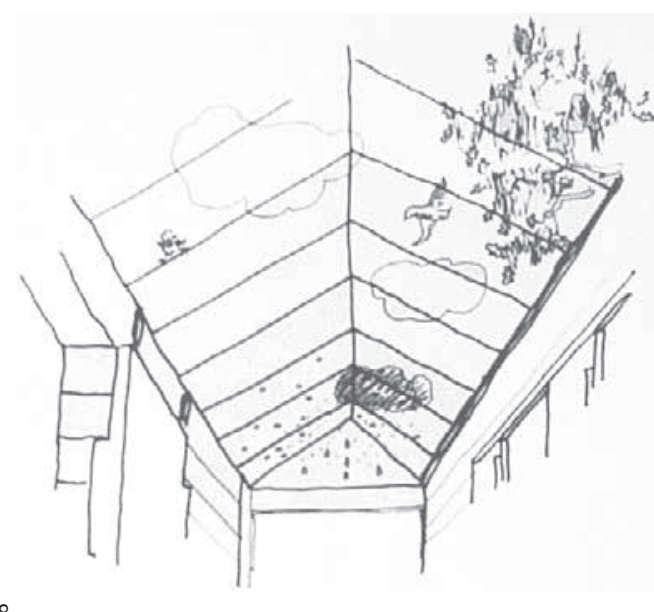

como lugares lúdicos equivalentes a los existentes en los entomos de los barrios obreros de la ciudad industria. La sensibilidad psicogeografica desplegada en el proyecto es capaz de hacer converger en una almóstera untaria' varios segmentos diferenciados del colegio que son manipulados libremente. Los giros y desplazamientos en la traza de esos elementos, permiten incrementar los accesos induciendo un flujo naturaly continuo entre interior exterior. La multiplicación de las opciones de uso potencia un mecanismo de apropiación, mediante la dación de posibilidades de elección y variación, y genera una mayor relexión en una movilidad que traslada la complejildad propia de la ciudad a un entomo caracteristicamente suburbano. El gradiente de intimidad reaparece como objetivo prioritario de proyecto. Los Smithson elaboran con similar cuidado que en Hallfield el rango y variedad del modo en el que los cerramientos y las ventanas se disponen en cada espacio; pero realizan una aportación nueva interesante con la inclusión de superficies acristaladas inclinadas en la cubierta de las acrecentadas áreas de circulación que elaboran una nueva calldad de ámbito a medio camino entre el dentro y el afuera (figura 8). En ese espacio, el cuerpo se proyecta hacia el exterior deste climatizado en el que los niños pueden continuar sus brutalistas se enfrentaban al diseño de los colegios de

actividades de modo permanente. Su cualidad permite la ciones que la climatologia produce sobre la arquitectura yla natura leza, tomentando la asimilación del lugar como . La adopción por pane de los Snithson de la concepción de la arquitectura como un todo "coordinado de cada una de las partes que constituyen un edificio" responde a una operaliva proyectual pragmática y adaptativa, pero es tambien el desarrollo de su 'orden conglomerado como respuesta a la fragmentación de la industria edificatoria. Existe un estuerzo por trabajar los materiales, a traves de su elección particularizada y evitando su enmascaramiento, como elementos sintácticos que, en su rellejo de la naturaleza constructiva del edficio, permiten al niño la comprensión profunda del entorno en el que desenvuelve su actividad. Los espacios vacios de Hunstanton folografiados como testimonio de una obra de arte ediricada se tornan en este colegio de

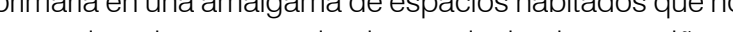
cuesta imaginar caracterizados y colonizados por niños. EDUCANDO ADOLESCENTES

Si el contrapunto al modo en que los arquitectos nuevo

19. SMTTHSON, AyP, op. cit., supra, nota 17, p.45. 


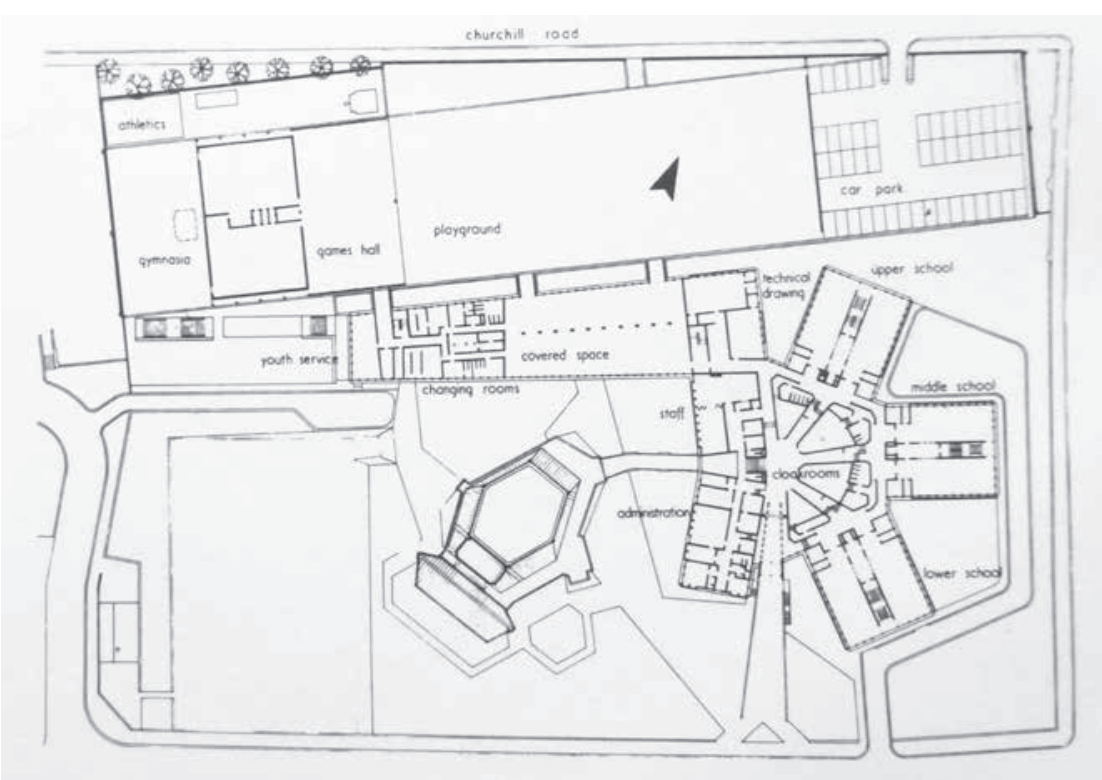

9. Planta de acceso del colegio Acland Burghley, con el desplegado de piezas fragmentadas y diversas
articuladas en un conjunto coherente. Sección trans. versal por la plataforma sobre las vías ferroviarias, el bloque de tres alturas y el auditorio 10. Amplio acceso de entrada entre la macla de volúmenes a modo de pasarela con el 'cluster' de tres torres a la derecha.

11. Vista del Colegio de Pimlico desde la esquina su-

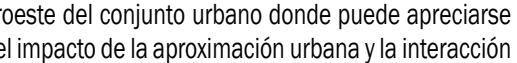
de los viandantes con la actividad del Colegio. La pis-

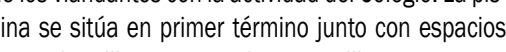
recreativos libres anexos de acceso libre.

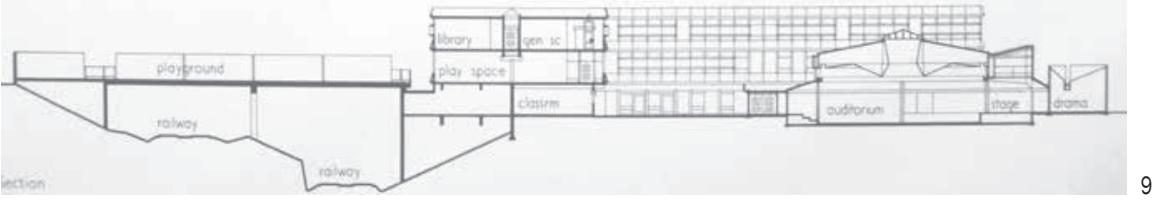

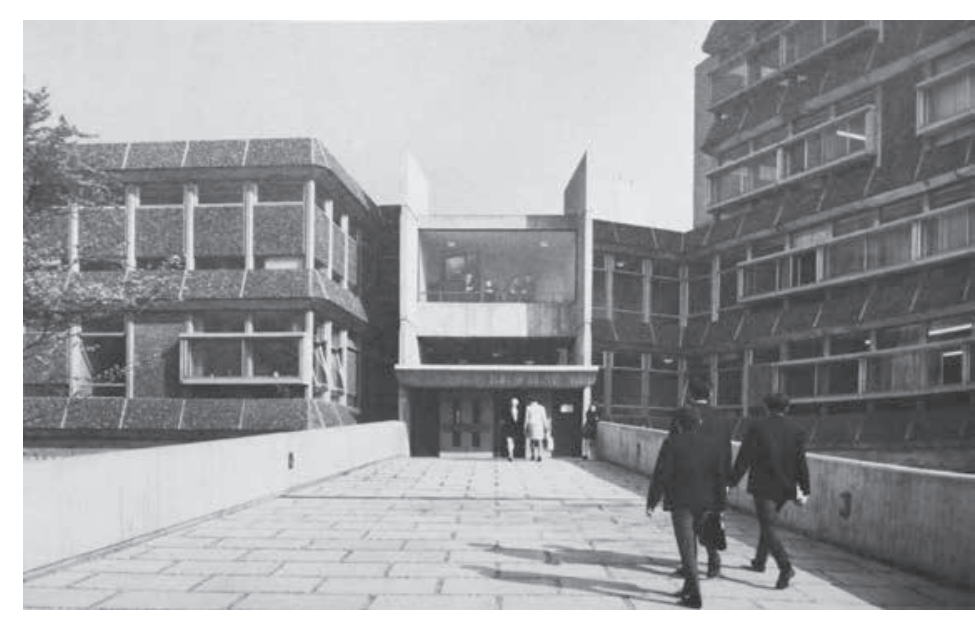

a través de patios internos o grandes ventanales, lo que fomenta la percepción de hiperconexión entre piezas difomenta la percepción de hiperconexión entre
versas aisladas dentro del conjunto unitario.

El empleo de una geometría conscientemente no orEl empleo de una geometria consclentemente no or togonal busca reforzar la sensacion de que el movimiento en el ínterior del colloglo se ínstaure cono un deventr

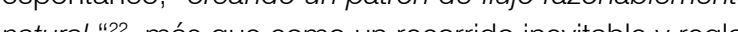
do. Estén ńnsis en la elaboración de la moviliad diversa y su experiencia como latiolad de Hellfiel un elemento recurrente en la arquitectura nuevo brutalista que se consolidó como rechazo profundo 'corredor institucional', senunciado por Howell, Killick y 'Partidge conto un mal frecuente y endémico de much Partidge como un mach

. risticas del programa son las generadoras de las formas del edifio en contunción con las particularidades de Los arquitectos operan en creando una plataforma so-

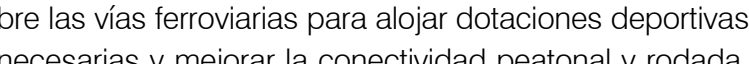
El coniunto se compone de diversas piezas fácilmente reconocibles de morfología y tamaño distinto según las actividades que acogen, las cuales quedan enlazadas por una circulación múttiple y prol'fica en sus cualidades ed ficando la revitectura como pieza que homotetica, entre construye las diversas escalas de la ciudad.

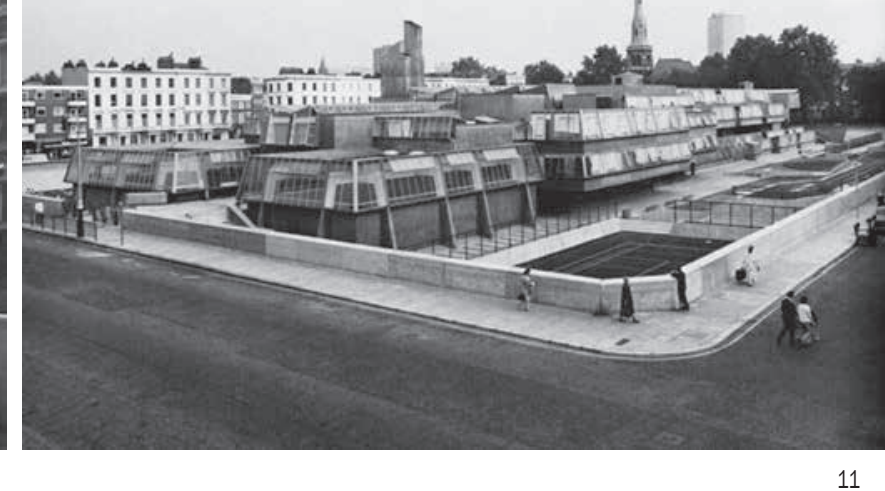

La entrada principal del Colegio Aucland Burghley escenifica la máxima prodigada por el estudio de arquitectos de que "un ma prodigadio no por el estudio de arquique debe ser percibido como accesible"24. Las entradas atraviesan bloques mavios que se despliogan invindo inequivocamente a su traspaso (figura 10). Esta prome nade en elacceso se continúa en l paso protegido ocasional aue transoure bajo los edificios, y secomplementa al interior con una separacín de las funciones a traves de pequeñs desnives como limites invisibles delimita dores de espacios en total continuidad. Todo ello, unido cubiertas que vivifica les superficies y las a laja de su per cepcín como material inerte dibuja una nueva geograft social construida con intención de aportar variedad e intensidad dentro del ámbito educativo.

El Colegio de Secundaria en Pimlico

Otro ejemplo insigne del Nuevo Brutalismo y un exponente destacado dentro del campo de la arquitectura que recibió el premio del Royal Institute of British Architects en 1972. lo constituye el Colegio de Secundaria de Pimlco. Su arquitecto, John Bancroft25, encuentra las claves de las decisiones más importantes que lideran el desarrollo del proyecto sacando partido a las características particulares del contexto en el que trabaja Reutiliza las cimentaciones de la edificación previa demolida y decide rehundir el total de la manzana (figura 11) de manera que

\section{2. íbid, p. 97.}

23. Í íbid, p. 98.

25. El colegio afrontaba la reubicación de 1750 alumnos procedentes de tres escuelas en un solar de escasas dimensiones en Westminster. Su diseño estaba en manos del London County Council, bajo el mando de Hubbert Bennet. Tias primeras tentativas de edfiticar el colegio como torre para liberar máximo espacio libre, John Bancroft, al cargo directo del diseño, encuentra una solución más económica y satisfactoria en una estudiada compactación e imbricacio 


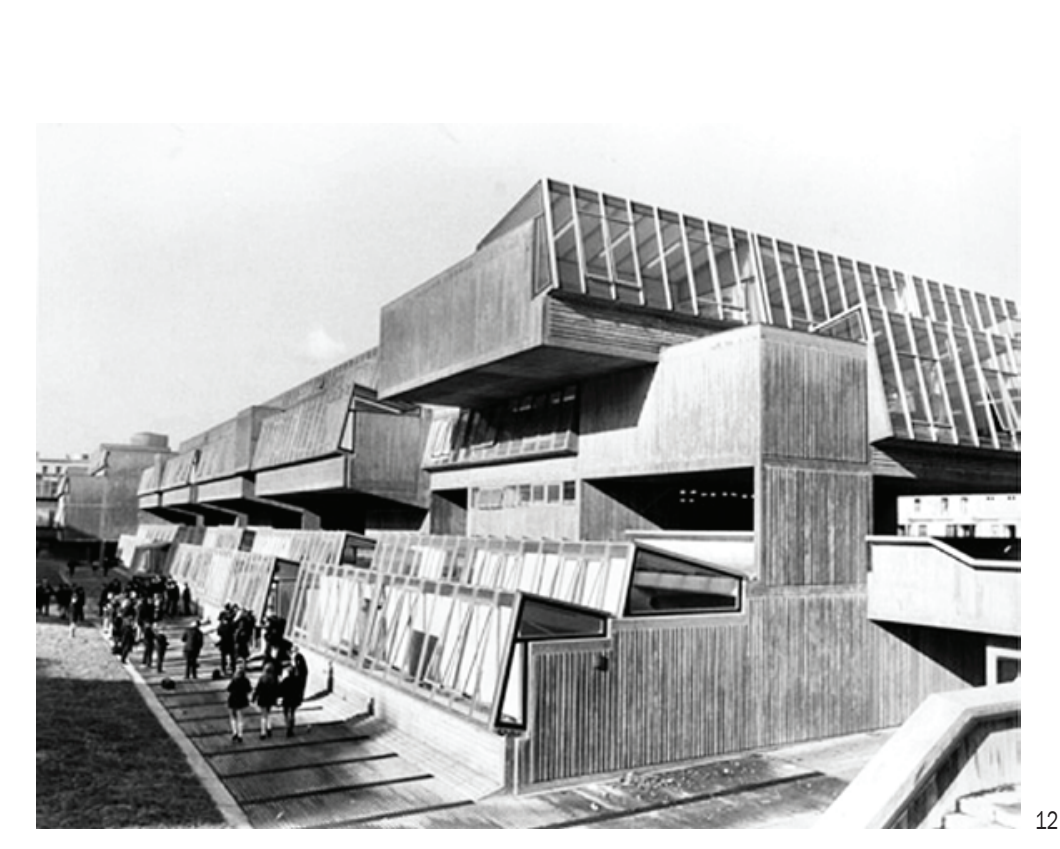

la altura final del edificio escolar con respecto a las colindantes resulta moderada y las áreas de recreo quedan también parcialmente protegidas del viento.

El colegio de Pimlico resuelve la pretensión de encontrar nuevas categorias de espacios urbanos posibles para el desarrollo de una espacialidad urbana intensificada ${ }^{26}$. Produce una interesante reformulación sofisticada de la condición demandada del uso del espacio publico y de la calle para el juego y la estancia de los niños en condiciones de seguridad y de familiaridad. Desde una cierta distancia, el nivel del suelo con las dotaciones exteriores queda oculto a los ojos de los viandantes, pero situados unto al murete penmerial que delinila el contorno del solar, el urbanita, el vecino, el familiar, puede observar parcialmente la vida que acontece dentro del ámbito del colegio. Esta cualldad dual que discure entre lo público y lo privado tiene traslación en el espacio exterior en Pimlico La percepción desde dentro reproduce una impresión de intimidad que termina por mitigar, en gran parte, la posibilidad de interacción visual anónima y casual otorgada a los viandantes desde arriba. Pequenos relieves, vallas diseminadas y topografías que surgen a nivel del suelo (figura 12) ahondan en la generación de esa sensación de límites psicologicos buscada por el arquitecto por beneficiosa para la formación de la personalidad de los dolescentes, tan reacios al control permanente.

El interior del Colegio maneja también un

con el recurso de la estratificación. En la cota rehundida de planta baja se ubican las funciones más públicas (figura 13) y destinadas a un aforo más amplio que disponen de escaleras de acceso libre y directo. Desde este nivel de entrada, las circulaciones se internalizan dentro del colegio y van disminuyendo en cuanto a su entidad su espacio asociado segregando progresivamente lujo de usuarios hasta los ultimos recintos superiores. Otras entradas independientes que conectan a diversos niveles aportan la posibilidad de desligar usos concretos, de compatibilizar distintos horarios, de coordinar diferenes tipos de flujo y usuarios, así como proporcionar una inmediatez de llegada.

La situación al nivel de planta primera de los accesos principales que enlazan el colegio en sus extremos con as calles circundantes, provocan la sensación de estar caminando por encima de un pequeño barrio o microcosmos. Un tránsilo a través de plataformas que discurre entre una sucesion de lucenarios cuyos recintos quedan aislados del tránsito libre circundante en planta baja po gruesos cerramientos opacos para obtener intimidad (figura 14). Este diälogo entre privacidad y conexion se consigue igualmente en las aulas docentes, que mantienen la sensación y funcionalidad del recinto de una clase pero se abren decididamente a la luz y a la contemplación del cielo, en un modo equivalente a los pasillos en el Colegio de Wokingham perogenerando, en esta ocasión una curiosa sensación de ingravidez sobre el vacío.

26. “...el colegio aparece verdaderamente como ha sido descrito: ' ‘n barco en un puerto seco'. Se relaciona muy bien con su entorno. El liseño urbano siem-
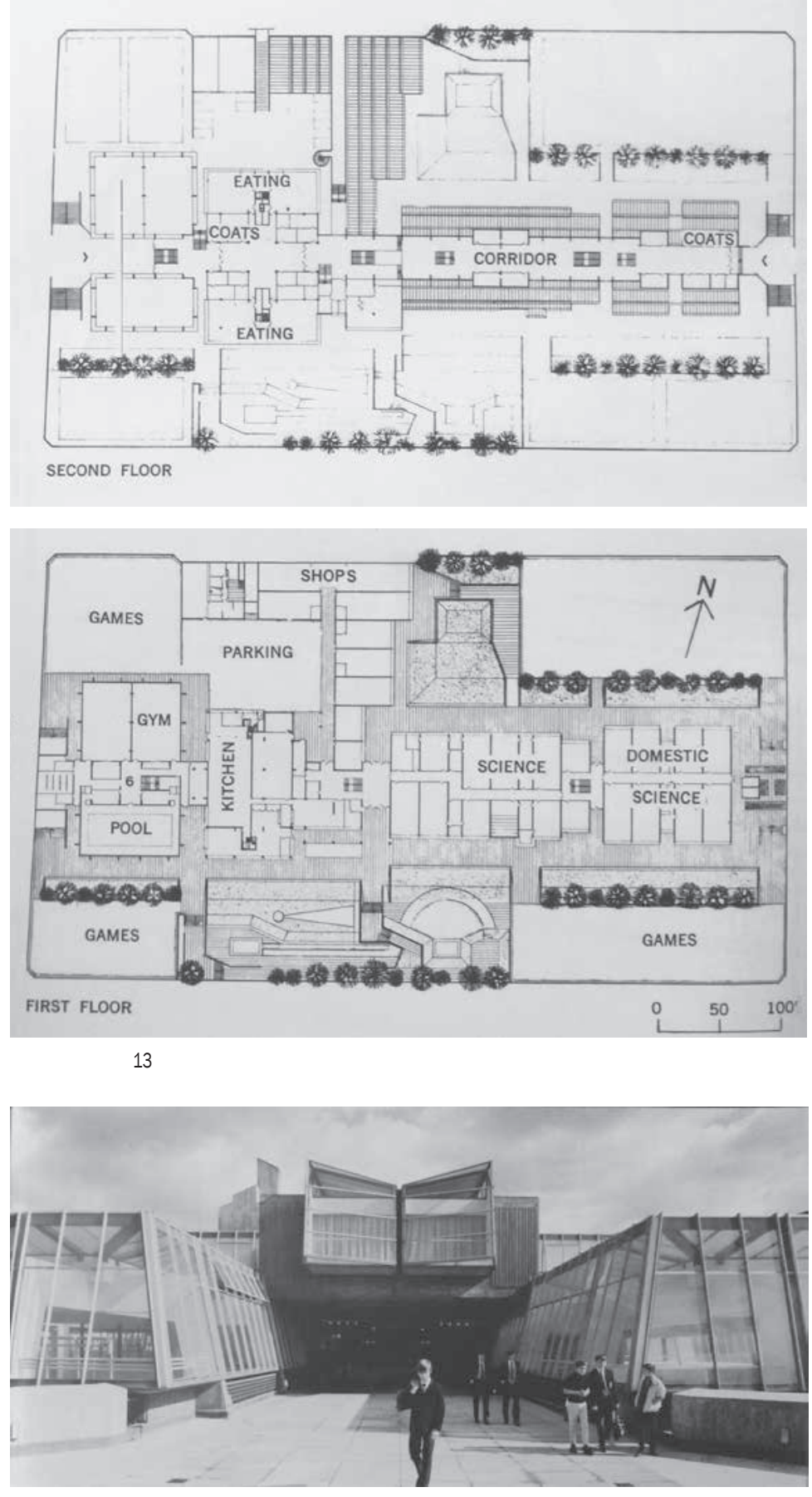

Las entradas al colegio transcurren entre robusto volúmenes evitando la colocación de una simple puerla rebajada en el lienzo de fachada. Masas recortadas acogen al usuario y provocan la dilatación perceptiva y emporal del propio hecho del traspaso creando un ámbito de acogida y estancia a modo de pequeña plaza en el acceso. El nivel de recepción del colegio en planta primera opera como un área de velocidad variable y un eje estructurador que aporta claridad al esquema ordenando una geografía tridimensional compleja.

El manejo de las superficies que Bancroft realiza busca aportar una "suficiente compleilidad que equilibre la gran masa"27 de la terraces colindantes. Pero, además, los recortes y desdoblamientos de las superficies obedecen a la voluntad de caracterización de los espacios interiores. La calle interna parte en dos como una hendidura profunda el volumen del colegio, recordando al proyecto para el concurso de la ampliación de la Universidad de Sheffield de Alison y Peter Smithson, y de ella emergen superiormente las aulas en voladizo mientras que a sus pies se dispersan maclas vidriosas y paños de hormigón afilados. Los recortes transversales marcan con la claridad que aporta su oscuridad intensa las entradas a recinto educativo. En definitiva, las superficies crean una piel articulada volumetricamente cuyos contornos pronunciados, reforzados por el juego de luces y sombras, construyen una fachada de una profundidad inusual donde puede leerse la ubicación de cada parte del programa. Los espacios de circulación y su caracterización dentro del colegio de Pimlico contribuyen a generar un buen clima que refuerza el estudio y motiva la asistencia, al tiempo que fomenta las relaciones sociales ${ }^{20}$. Las entradas principales que conectan longludinalmente colegio con las calles colindantes estimulan un transito mas continuo y favorecen el surgimiento del saludo. Atraviesan el total del edificio y adquieren unas dimensiones amplificadas a modo de calle interior (figura 13) construyendo un espacio de relación que opera como umbra semipúblico al que los pasillos de acceso a las aulas de 27. Íden

28. John Bancrofft expresaba su convicción en la incidencia que la arguitectura podía tener en el buen funcionamiento del colegio, señalando la importancia de la adecuada organización en planta y yatención a necessidades concretas. Ílem. 
destacado que reconfigura usos y recorridos. Con su en el contexto del nihilismo imperante actualmente, pero lestimonio contorman uno de los ultimos movimientos de tal vez un objetivo que conviene rescatar ante la falta de resistencia que aún contia en el potencial de la arquilec- ideas de hacia dóndo vamos, porque como Reiner de lura para inducir comportamientos y liderar camblos que Graff señala, "Una sobredosis de utopia es peligrosa que se inserta. Tal vez una visión poco realista encuadrada utopía que puede ser igualmente peligrosa" 31 .

\section{Bibliografía citada:}

BANHAM, Reyner, “The New Brutalism”. En: The Architectural Review, 1955, diciembre, pp.354-361.

BANHAM, Reyner "City as Scrambled Egrg" En: Cambridge Opinion. 1959, "Living with the 60s" edition, n.17, pp.18-23.

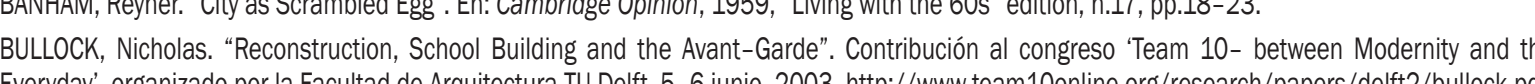

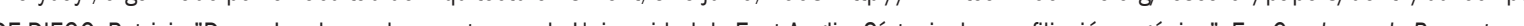

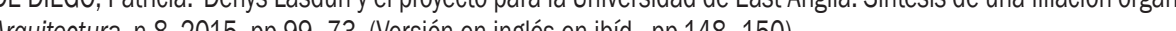

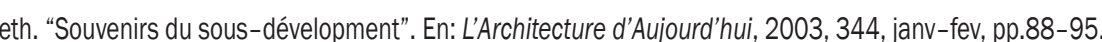

FREARSON, Amy. "An underdose of utopia can be as dangerous as an overdose". En: Dezeen, 12 de septiembre 2012. Http://www.dezeen

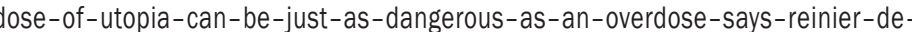

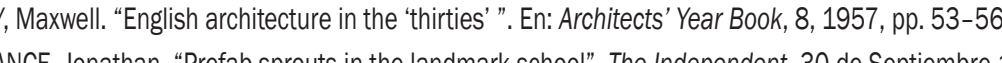

(P) thes-sprout-in-the-landmark-school-1356220.htm.

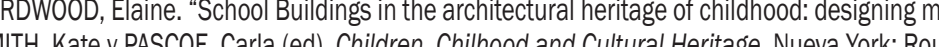

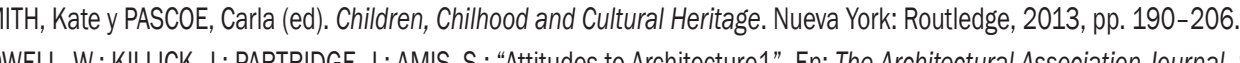

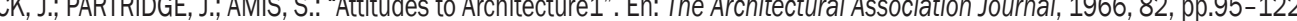

KNABB, Ken (ed): Situationist International Anthology. Berkley: Bureau of Public Secrets, 1995.

LLEWELYN-DAVIES, Richard y WEEKS, John. “Endless Architecture”. En: The Architectural Association Journal, 1951, Julio, pp.106-112.

LYNCH, Kevin. “The Form of the Cities”. En: Scientific American, 190, n.4, pp.54-63.

YNCH, Kevin. The Image of the City. Massachusets, Londres: MIT Press, 1960.

SHARIATMADARI, David. “60 seconds with....John Bancroft". En: Building Design, 5 de marzo de 2008. http://www.bdonline.co.uk/60-secondstoft/3108135.article.

SMITHSON, Alison. "Soho House". En: Architectural Design, 1953, diciembre, p. 342

SMITHSON, Alison Y Peter. Italian Thoushts Londres: A\&P Smithson, 1993.

SMITHSON, Alison y Peter. “A Cluster city" a new shape for the community" En: The Architectural Review, 1957, noviembre, pp 333-336

SMITHSON, Peter. “The idea of architecture in the '50S' '. En: The Architects' Journal, 21 de enero de 1960, pp.121-126, STADLER, Laurent. “New Brutalism','Topology' ' and "Image'. Some remarks on the architectural debates in England around 1950". En: The Journal of
Architecture, volume 13, number 3, 2008, pp.263-281.

wooD, Denis. "Lynch Debord: About two Psychogeograhies". En: Cartographica, v.45, 3, pp. 185-200.

Patricia de Diego Ruiz (Madrid, 1975) Prof. Asociada de Proyectos Arquitectonicos -EISA-UAH. Beca F.P.U. en la ETSAM-UPM

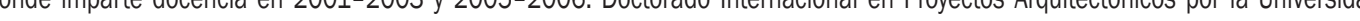
Politécnica de Madrid "Entre tradicion y transición. Genessis y cambio en la arquitectura del Nuevo Brutalismo". Becas y Estancias Internacionales de investigación en Gran Bretaña. Miembro del Grupo de Investigación Paisaje Cultural de la U.P.M. con participación en proyecto compentiso de 
BRUTALISMOS EDUCATIVOS. LA ARQUITECTURA COMO NUEVA PSICOGEOGRAFÍA SOCIAL Patricia de Diego Ruiz

p.85 INTRODUCTION

Architecture can hardly obviate the impact that it causes, more or less decisively depending on the case, on the and material defining characteristics, condition human beings in their functional development and their specific way of understanding their environment. A building and its closest group of architecture define specific and distinguishing spaces, consciously or not, but implying anyway a unique understanding of the ambience. This immanent capacity of architecture gains importance when dealing with educational buildings and schools. Responsibility grows as incipien The Great Britin of midtest century in the dect

cades after the end of the Second World War, marks an interesting discover the interactions that educational and political guidelines can have on their architectonic transposition. The primacy of some objectives above others as well as the hierarchical order in the decision-making
process, decisively determine the architectural design approach and the resources handled by architects in schoo architecture and its scope

The most renowned and studied case in this geographical and temporal context is that of the Hertfordshire Prefabricated Schools. Designed by David Medd along with the Hills company, from the beginning they were an outstanding example of the moment recognised for their evident achievement in providing two hundred schools in a
period of fifteen years, moreover, on a pretty tight budget. However, the Hertfordshire Schools managed to introduce an ontological dimensional change of a much greater scope in British architectonic debate, which is not so we known. Nicholas Bullock points out how John Summerson identified school buildings as "a tendency to go in search of principles (...) principles announced as building"1 and J.M. Richards maintained them as "defining the face of a new
and especially English architecture". They were the start of a new architectonic approach favoured by the political and public sphere in which engineers and builders worked in a collaborative way with architects in the planning of structura

p.86 systems that could be used as pre-solutions on a large scale. The pecullarty of tits conception as a complete mesh meccano, based on the eight feet by three inches module, meant that not only classrooms could be prefabricated and numerous combinations in particular solutions (Figure 1), thus meeting the demands of British eduectitanalists The Hertfordshire Schools were initially admired by the 'angry young generation' as well as by the most avantgarde architects of the previous generation who supported the British theoretical and practical revolution that would be led by the New Brutalism?. But soon the emerging enthusiasm with the pragmatic and syntactic values of this new a restrictive way by these architects, Its approach was considered insufficient to be able to adiust to real complex social demands, and even perverse, for speed and economy were perpetuated in them as priority objectives to which architectural form was subordinated. Typification prevailed over the best specific architectural solution thinking. In the search for principles so accurately figured out by Summerson and that was so crucial to the new generation of architects, industrinalisation turned trom being the only answer to being an important, naturally-assumed agent.
The system as a general solution gave way to customised prefabrication and the introduction of traditional low-cos techniques in order to compete in economical terms. Then, the focus of interest in the field of architectural creation moved towards urban theories. Their reflections led the way in the opening of innovative manners trying to understand the environment and to redesign it in contemporary terms to the detriment of a disappointing C.I.A.M. that distanced itself from its proposed and visionary perspective, while also falling to understand the relationship between the form

ell known in the British avant-garde circle, not only in its European development shared the significance attributed in these urban theerican context supported by Kevin Lynch. The Brutalist sensitivity characteristic of the mechanised era and a typical feature of a socieity in constant change. It would focus on the potential of pedestrian traffic, assuming it as a device for registering and knowing the environment; but it would als

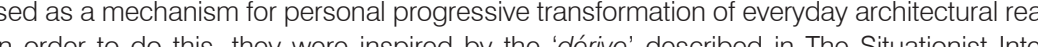

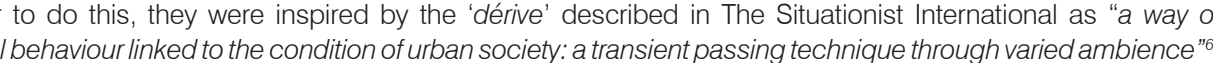
Each of these 'ambiance' corresponded to parts of the city emanating a special force as ambience that led to the

p.87 development of a sentiment or mood associated to its character, but which also referred to the geographical condition
itself as small neighbourhoods or urban fragments. Denis Wood points out the parallelism between The Situationists and Lynch, not only in their defence of the necessity to walk the city, but also in their common interest in the 'unitités
Satisfaction', stated "We are concerned here with the psychological and sensual effects of the physical form of the determinant elements in urban environment perception making a detailes he would ceepen in the study of the most passages and all kind of ornamental details and distinctive elements such as iron gates, balconies, bow-windows or entrance landings. This attention to unevenness and protrusions on façades would have a similarity with the use of projections, folds and complexity given to the architectural cladding of Brutalist schools, which work as signs that mark, brighten up and illuminate the dally domestic ambient. The distancing from abstract material inishes and their through a perceivable aspect deriving from the articulation of particularisised forms; forms that arise from the previous
the meticulous analysis of the array of internal necessities and the enhancement of their distinctive features. In this sense, this approach is inspired by Lynch's work, whose effort to understand mental images conceived by American city

Inhabitants was based on the study of the characteristic value of visual form?
The word 'image' was taken from the urban context and related to architecture by Reyner Banham in his article has derived in the general interpretation of its most literal translation as a sculptural and visually-astonishing quality. Laurent Stadler has, nonetheless, highlighted the operative condition of the use of the concept revealing various ramifications that such a complex and polysemic term had in the fifities' debate $e^{10}$. Banham's analysis, in its architectural scope, clarified that "the building should be an immediately apprehensible visual entity; and that the form grasped by the eye should be conimmed by the experience of the builaing in use" "I. It is the essence of image as a configuration ransform it into a mental internalised image, the one that fits in with the concept of experiential environment studied and claimed by Psychogeography.

EDUCATING CHILDREN

作 Bulletin no1 published by the Education Ministry in 1949. The partial relaxation of this quideline in the education nexorably followed the bulletin content, the architectural result turned out to be unusually far from its traditional archelype.

Wlfield School started to be planned in 1950 and although it is not commonly related to the New Brutalism'2, its Iriendly radicalism set the corner stone that proclaims the right to search for contemporary forms and establishes a shift of paradigm that breaks as much with British 'modern' orthodoxy as with the state venture for industrialised school architecture e $^{13}$. That flexible and open arrangement admired in the Hertfordshire Schools that proposed buildings as
a system of modular parts, transcendentally mutates into an architectural layout as a structure of relationships, as a heteromorphic (Figure 2) and varied organismit. The kaleidoscope of forms unfolding in the project is not contrary to
the attention paid to repetition as a characteristic of the Second Machine Age arranging the infant area as a cluster 15 where all cells have the same volume, coinciding with the whole classroom with a strong paralle with the procedure $p .89$ existing in industrialised schools. But that repetition of form does not arise from technical constructive impositions nor does it follow a previous system. Lasdun and Drake propose a specific pentagonal shape that not only allows varied groupings in bigger units, but which derives from the search for the best 'capable form' to harbour multiple internal Constructive and material techniques are combined freely in a non-eclectic, unprejudiced spirtit while prefabrication. which plays a principal role in the school construction, is not apolied homogenously and in a unitany manner to the whole. Its employment adjusts to the functional characteristics of each of its areas and starts to be used as an eloquent resource to serve the dominant form providing each part with a distinctive character.

Form arises not only from intemal deterninanc factors, but is equally senstive to external significant forces, in the field of architecture, with a focus on pre-existences. The Hallfield School assumes the axes common to the general
layout of residential blocks among which it is inserted to make them fit in with them in the general layout of the school building plan (Figure 2), including a new third diagonal axis that distorts in reality the monolithic, axial and regular perception that might be held of the school.

Compelled by the considerableelevaltonot heresesidentialiblockssurroundingtheschool, Lasdunand Drake implement Igeneratingaprogressiveprivacygradientifromthebackaccessstreettowardsthegardenontowhichtheschoolopens (Figure
4) The work with diminishing volumes and a high sensitivity when dealing with wall facings and openings achieve particular levels of intimacy and provide playing areas for children, which aim at retaining a certain self-absorbed nature (Figure 5) 
he display of particularization that this school holds towards the diversity of use and needs required by each age group is made possible, to a great extent, due to the use of circulation as a weaving element. Environmental units
of singular form and quality clearly appreciable on a perceptive level bound together by a passable magma. Crossing spaces transmute from being inevitable residual elements that eive access to various compassable magma. Crossing spaces that add an intrinsic value that substantially enhances the building

resting spaces and stairs, along with the specific manner in which each of these circulation spaces relates with the inward and outward gaze, turn the network of corridors created for the school into dynamic and free-flowing phenomenological and spatial pulsation. The proliferation of connections with the immediate environment at differen

It is gradifually revealed in sequencees alternating dynamic areas and static focal points that reinforce the creation of 'ambiances' producing mental images that help recognise and assimilate space. Soft turns in the geometry of the plan as well as changes in the opactiy of enclosures demonsitrate the architects aim to design an amusing and unexpected are elongated circulations, but prolific in characteristics that build learning and mobility-stimulating environments.

Wokingham Primary School

The influence of Lasdun and Drake's school on Alison and Peter Smithson's approach to architectural projects is
shown in the shitt shown in the shitt from a disciplined geometry, perceptively compact and regular, which is displayed in their famous 1958. The mobility parameter as dynamic generation of the architectural layout is maintained through the use of circulation as the structuring element along which differential and particular pieces connect. A programmatic package consisting of a classroom and its linked service spaces form a small sub community that repeats itself in series and ritylimically along an irregular path that resembles a growing spiral directrix (Figure 7). This project is, according to Kenneth Frampton, "the Smithsons' socio-cultural 'cluster' at it most elegant

The use of open-ended torms that are 'make-places w goes along with the variation of paradigm permitting Wokingham School, through its non-Euclidian geometry and through its implementation, to fold and mould the space child perception and development by the Smithson. Its employment on exteriors through the delimitation of paths photographs taken by their friend Nigel Henderson in Bethnal Green ${ }^{18}$ Classrooms face south and can use these

p.91 outdoor areas as open-air non-regulated educational spaces or as play areas equivalent to those existing in the surroundings of industrial working-class neighbourhoods.

The psychogeographical sensitivity displayed in the project is capable of gathering in a 'unitary atmosphere' various differentiated segments that are arranged freely. Changes of direction and displacements in the layout of
these elements increase the number of accesses that prompt a natural and continuous flow between interior and these elementerior. The proliferation of usage options favours a mechanism of appropriation, providing possibilities of choice and variation; it also generates greater reflection in a circulation that transfers the city's idiosyncratic complexity to a characteristic suburban environment

A gradent o mintacy reappears as a priority goal in the project. The Smithsons devise with analogous care to tha in Hallifield the scope and variety with which closures and windows are placed in each space. But they also make a
new interesting contribution with the inclusion of sloping glazed surfaces on the roof of the increased circulation areas that produce a new quality atmosphere halfway between the inside and the outside (Figure 8). In this space, the body projects itself outwards from a psychologically-safe and technically well-tempered environment where children can carry on with their activities on a permanent basis. Its essence allows to raise awareness on the passing of time and the climatic variations produced by the weather on architecture and natre ens The Smithsons' adoption of the conception of architecture as a

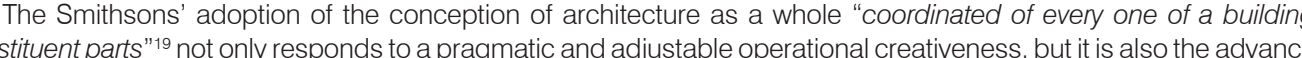
of their "conglomerate ordering" as a response to building industry fragmentation. There is an effort to work with materials through their particicular choice and avoid masking them, as syntactic elements that, in their reflection of the their activities Hunstanton's pure and empty spaces photographed as testimony to an edified work of art th this cary ou school turn into a patchwork of inhabited spaces that one can easily imagine characterised and colonised by children.

EDUCATING ADOLESCENTS

p.92 If the counterpoint to how New Brutalist architects dealt with primary school design was the standardization of the light and flexible building system created by David Medd evolved to be raised up to four floors and started to be (t) satisfactory plan organisation, which together with the more unruly nature of teenagers derived, in the opinion of Elaine

Acland Burghley Secondary School.

Of all secondary education centres, an outstanding case of innovation in floor plan arrangement, among other merits, for which it was awarded Grade II listing status, is the Acland Burghley School, built between 1963 and 1967 by William Howell, John Kilck, John Partidge and Stanley Amis. Ils organisational structure denoles its authors" internalised subdivisions and seek the fragmentation of the school shape in various cellls like small neighbourhoods ' 'mith interinal in psychogeographical terms, (Figure 9), whose parts remain linked by the development of a circulation that recalls a civic status. ${ }^{21}$

The missed and lost social condition of pre-modern city public and dalily spaces is emulated through the design of a circulation system transcending the functional aspect and intensifining its relational nature. The southern access 1o the three classroom towers 'cluster', with its star-shape ramifications from the widening of the meeting space
as a square, demonstrates this vocation. Transition occurs crossing different atmospheres and enhancing repeated and sequenced contact with external surroundings through internal courtyards or large windows, thus favouring the p.93 sensation of hyper connection between isolated pieces within a unitary complex

The use of a consciously non-orthogonal geometry aims to reinforce the sense that movement inside the school occurs as spontaneous progression," "creating a reasonably natural flow pattern"2, more than as an unavoidable and
regulated route This emphasis on the elaboration of a varied circulation and its experience as a series of articulated spaces, was since Hallfield a recurring element in New Brutalist architecture, which became established as a profofond rejection to the 'institutional corridor' denounced by Howell, Killick and Partridge as a frequent and endemic problem

of a lot of bureaucratic architecture 23
This school shows how the needs and features of the programme generate the shapes of the building in conjunction with the particularities of the surroundings in which it stands. The architects firstly operate at an urban level,
building a platform over the railway lines for the necessary sports facilities and to improve pedestrian and vehicular connectivity. Meanwhile, the complex is arranged in several easily recognisable pieces with different morphology and size in correspondence with the activities held in them. They are interconnected by a multiple circulation, also prolific in qualities, building architecture as a whole that assembles, in a homothetic way, diverse city scales

The main entrance to Acland Burghley School portrays the key principle praised by the architects firm that "a unfold unambiguously inviting us to enter (Figure 10). This promenade in the access has continuity in the occasional protected passage running under the buildings, while it is complemented inside with a functional separation through slight differences in level delimiting spaces in total continuity. This resource, together with the skifful use of projections on façades and roofs enlivening surfaces and distancing them from being perceived as inert mater

Pimlico Secondary School

Another famous example of the New Brutalism and an outstanding exponent in the field of architecture, which was awarded the Royal Institute of British Architects prize in 1972 , is the Secondary School in Pimlico. Its architect, John context in which he had to work Hor resed the foundations trom the previous demolished building and decicted to depress the whole block (Figure 11) so the final height of the school in relation to the surrounding buildings would be moderate and the playgrounds would also remain partially protected from the wind. Pimlico School resolves the aspiration to find new categories of urban spaces that make it possible to develop
and an intensified urban experienceer. If trepresents a sophisticated and interesting reformulation of the requirement to use
the public space and the street for play and the children's presence in safe and familiar conditions. From a certain ine public space and the street for play and the children's presence in safe and familiar conditions. From a certain
distance, the ground level with external facilities remains hidden to passers-by, but close to the perimeter wall fence bounding the site outtine city dwellers, neighours reltives, can partaly obsene life taking place inside the school This dual quality flowing between the public and the private is also transposed in the outside space in Pimlico. The perception from the inside reproduces a feeling of intimacy that finally reduces, to a great extent, the possibility of anonymous and casual visual interaction given to passers-by from above. Small slopes, scattered fences and

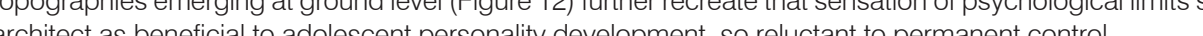
Temore public tunctions aimed at a wider capacity are placed in the depressed ground floor (Figure 13) and have direct and open

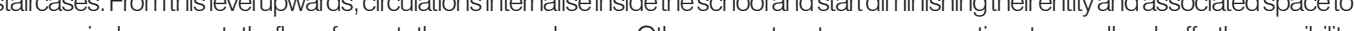

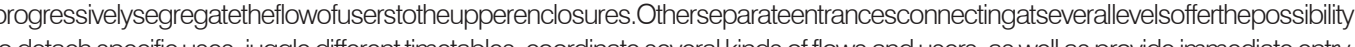


p.95 The architect evokes the sensation of walking above a small neighbourhood or micro cosmos by placing the main entrances that connect the school whis surfounaing streets on the first-lioor level. A perambulation along plattorms running between a series of skylights which reconcile visual exposure with intimacy isolating space below from free

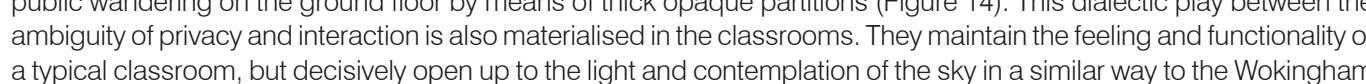
school corridors, but on this occasion generating a curious sensation of weightlessness over space.

School entrances unfold between robust volumes avoiding the placing of a simple door lowering the surface of the ext of passing, creating a small space for gathering and staying in the form of a small piazza. The school reception level
on the first floor acts as an area of variable speed and as a structuring axis that brings clarity to the layout ordering complex three-dimensional geography.

Bancroff's handling of surfaces aims at giving "enough complexity to balance the great bulk"z" of the terraces beside. Moreover, the silnouetting and unfolding of the architectural envelope arise from the characterisation of the internal spaces. The inner street cuts, like a deep fissure, the school volume into two pieces, recalling Alison and Peter
Smithson's project for the Sheffield University enlargement competition. Above the fissure, cantilevered classrooms emerge on the top while vitreous conglomerate and sharp concrete walls are dispersed at the bottom. Transversa cuts, with their intense obscurty, clearly mark the entrances to the premises. In short, surfaces create a volumetric articulated skin whose prominent contours, reinforced by the play of light and shadows, create an unusual deep façade where each part of the programme can be placed.

(t) create a friendly climate that longitudinally connecting the school with adiacent streets encourage a more continuous passage that favours spontaneous greeting. They cross the whole of the building and achieve amplified dimensions as an internal stree (Figure 13) conforming a relational space that acts as a semi-public threshold and towards which access corridors to

p.96 classrooms on upper floors appear as balconies allowing for the contemplation of the daily coming and going like an almost urban path (Figure 15)
Pimlico School was designed

政 to the city and to its potential use by residents and children that were not pupils. Attention to the social dimension took place in a higher degree of elaboration when including specific areas devised to enhance the formation of sub communities within the massive school capacity. Banctort designed eight big dining rooms that could also be used group. A facility thought as necessary to ensure the proper development of the 'scales of association'22 Flexibility of the auditorium equally demanded personal implication to choose and improvise a wide range of musical and drama performances, using, as in Hallfield, adjacent circulation space as a backstage area

CONCLUSIONS

The Hertfordshire Prefabricated Schools constituted an incentive for the search for principles through which post-war architecture could be updated, but soon, their commitment to speed and economy in detriment of form led to the emergence of a more adaptive and inclusive attitude that assimilated the industrialization, cost and technology as
instruments at the service of the dominant form; a form understood in its broadest dimension and unifying sense, as e and support of potentialities.

The understanding of architecture as a small part of a city by the Brutalists favoured, on the other hand, a correlative use of urban theories and mechanisms developed by The Situationists and Kevin Lynch in the architectural project. and circulation as a characteristic urban feature led to the emphasis of circulation as a new design parameter als governing the specific way to implement buildings. This generative dynamism articulates sequences of spaces, the organisation of which adjusts to the previous particularities of the context, and whose formal and material expression
derives ad hoc from the texture of social activities that take place in them. The significance of the plan from which the

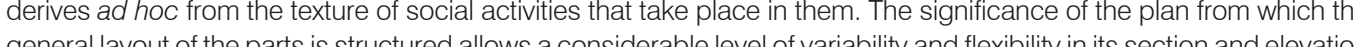

p.97 fitting, permitting the form to support each of the freely particularised spaces in a coherent and unitary ensemble. The Hallfield, Wokingham, Acland Burghley and Pimlico schools are proposed as structures developing the 'Cluster' and 'pattern' as models of thought interpreted in architectural terms as 'conglomerate orderings' and 'complex

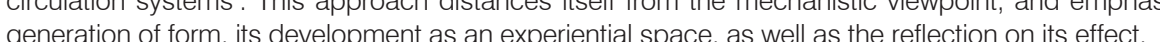
schools satisfied the social service commitment typical of the post-war era and encouraged the search for a singula architectural form according to the Zeitgeist of the moment. This allowed to place the child in the centre of the discipline

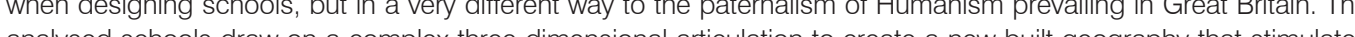

diverse scales of association and allows the identification of each 'community' with the part of architecture that it

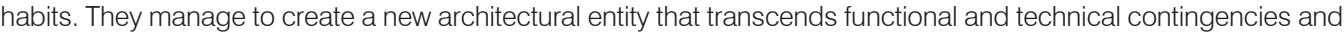
displays a new psychological reality raised through playtulu-constructive and spontaneous behaviour that takes place the child in its civic status as a social being that inhabits collectively.

This understanding of school architecture as a spatial-temporal experience implies that the notion of limit gains elevance. Its material and formal quality affects the body positioning in space and determines the emergence of certain activties against others. Its perceptive attribute as a more solid and built barrier or permeable and diluted interior, threshold and outside space. This occurs both from a visual perspective as well as from one of physical Iransposition

Accordingly to this perspective, formal heterogeneity of the envelope and variation of its textures, scales and atmospheres appear in Brutalist schools. Its purpose is to favour the development of human activities and to create social relation environments, besides defining moments of difrerentiation and particulansed expentence in the use communion and empathy take place with the surroundings.

CONTEMPORARY REFLECTIONS

In spite of the bad reputation attached to New Brutalism architecture, the schools studied in this article received lavourable review trom critics and a positive overall evaluation from its users30. Their project planning proposes on the awareness of the duty and responsiblity of school feclities to stimulate and shape the civic nature of future citizens through the creation of new inhabiited landscapes. Built environs which the user variably makes their own every day, following a non-univocal pattern of behaviour. Where polyvalence is a common resource and the inhabitant is a leading player that reconfigures uses and paths. Through their testimony, these schools define one of the last p.98 movements of resistance that stit believes in he polential of archtecture toinduce behaviours and corm mand changes that improve the physical surroundings and the society in which it is inserted. It is maybe an unrealistic view within the
framework of current prevaliling nihilism, but it may be a goal worth rescuing when faced with the lack of ideas about where are we going; because as Reiner de Graff notes, "An overdose of utopia is dangerous but architecture today is characterised by an underdose of utopia, which can be just as dangerous" 31 .

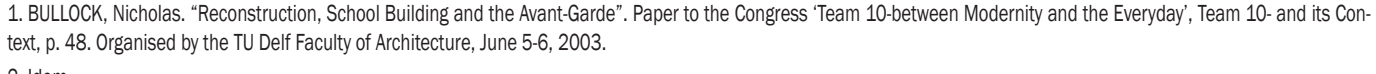

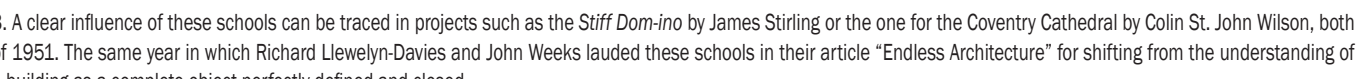
buildining as a complete object perfectly defined and closed.

W

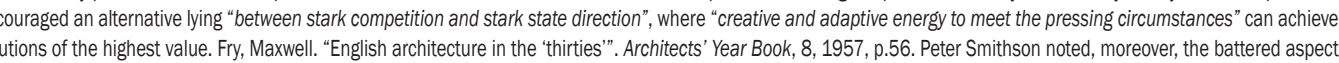

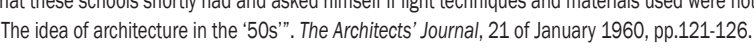

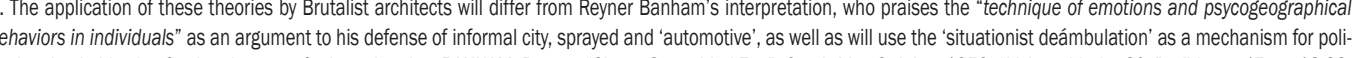

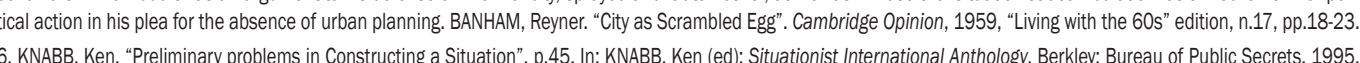

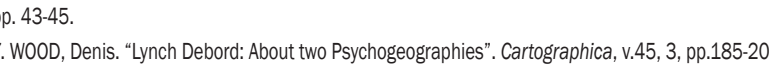

8. libi, p.190.

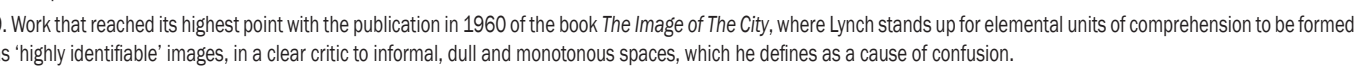

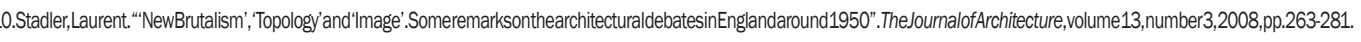

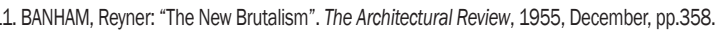

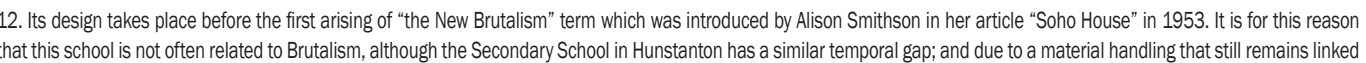
I received inheritance, its apperarance does not seem to fot with the strong aesthetics that is routiney yatributed to the movement

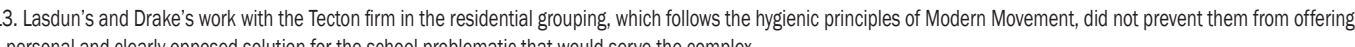

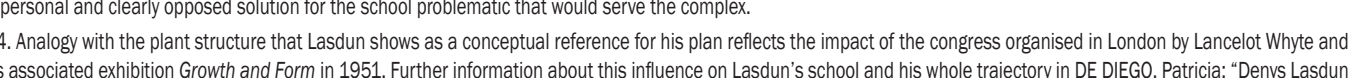

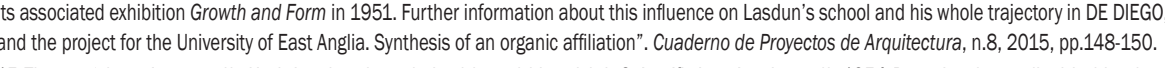

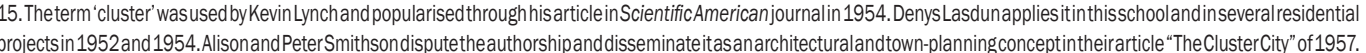




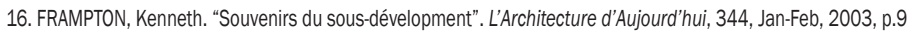

17. SMTTHSON, A\& P: ttalian Thoughts. London: AQP Smithon, 1993, p.43

(1)

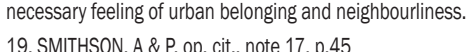

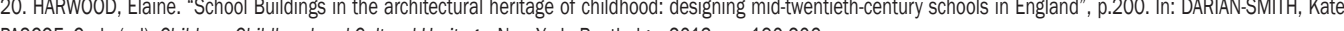

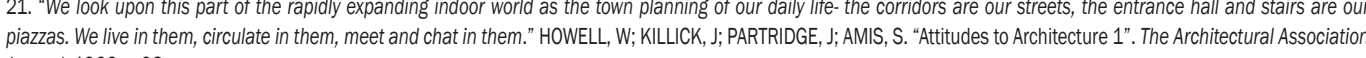
22.1 lid 0.097 .97

23. 1 lid,, .99

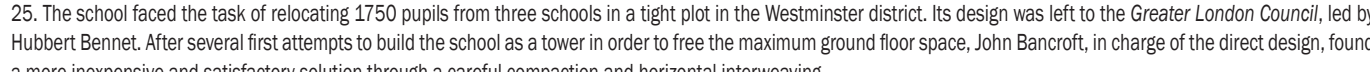

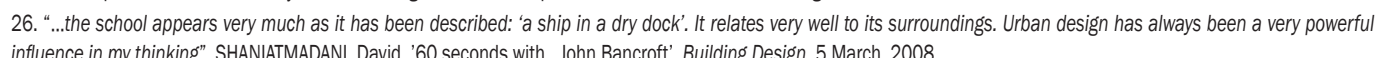

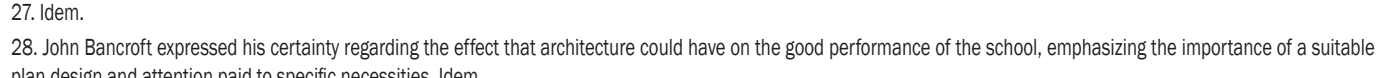

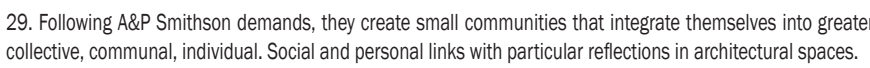

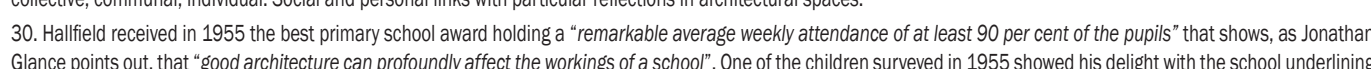
that " "once you get inside, you don't want to leave". The school has been working stood for more than sixty years and has been senstivively extended with Caruso St. John's

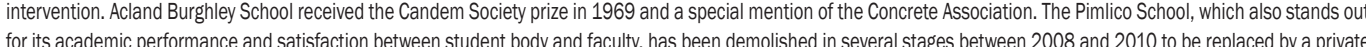

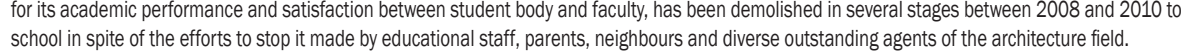

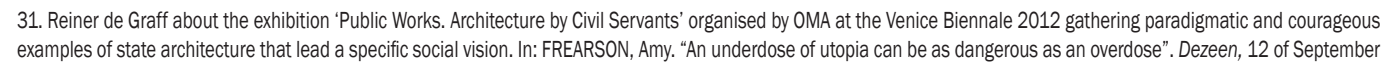




\section{Autor imagen y fuente bibliográfica de procedencia}

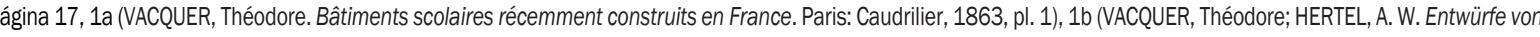

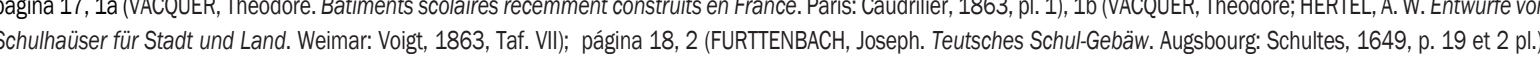

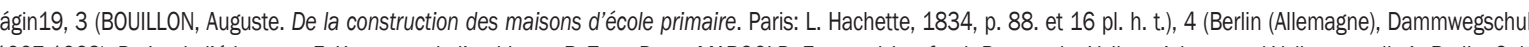

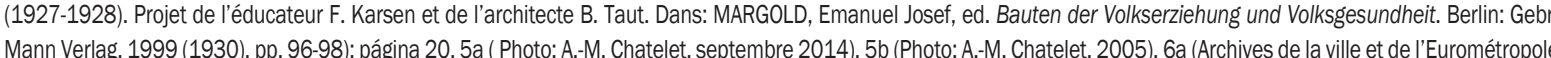
(1) Maquette de la grande percéé; E. Maechling, Musée historique de Strasbourg. Ophoto Musées de Strasbourg, M. Bertola), 7b (GOURLER, BIET, GRILLON et Feu TARDIEU.

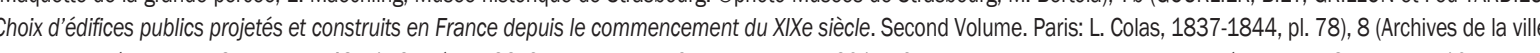

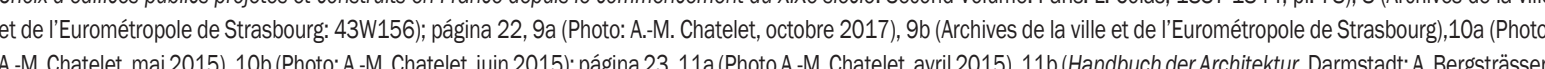

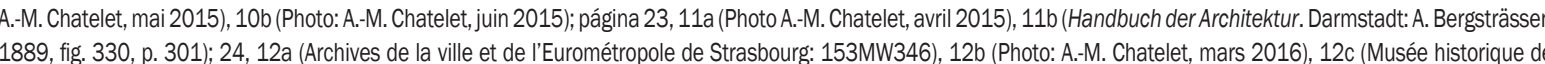

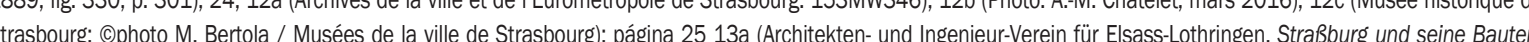
Strassburg: K. J. T. Trübner, 1894, p. 312), 136 (Photo: A.M. Chatelet, décembre 2013), 14a (Photo: A-M. Chatelete, avili 2016), 14 b (Photo: A.M. Chatelet, mai 2015); página

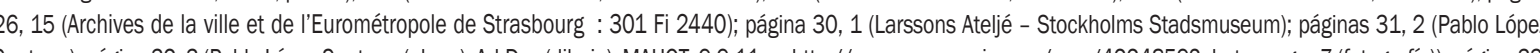

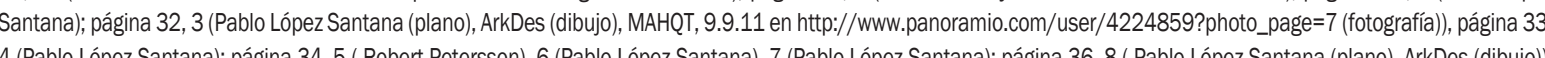

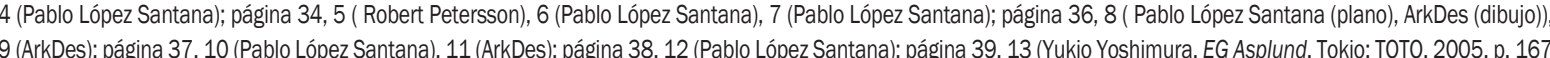

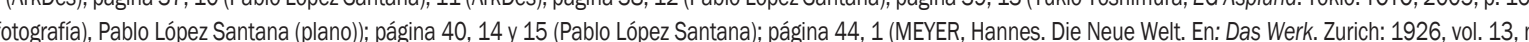
7, p. 218), 2 y 3 (KIEREN, Martin. Hannes Meyer. Dokumente zur Frühzeit Architektur - und Gestatungsversuche 1919 - 1927. Heiden: Niggl, 1990, p. 18; página 47, 4

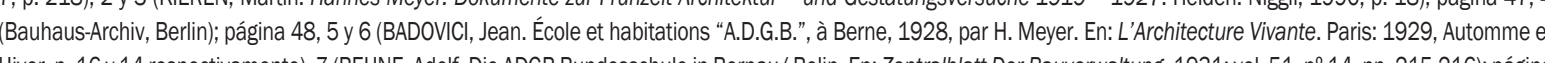

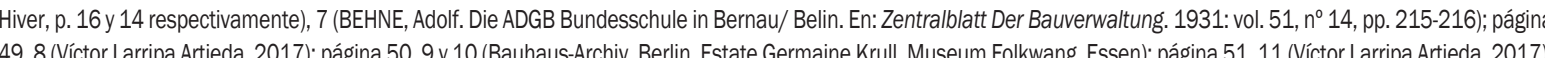

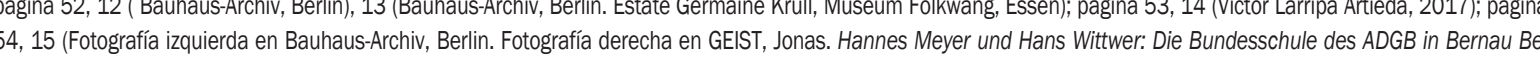
Berlin: 1930-1983. Potsdam: Potsdamer Verlags Buchnandlung, 1993, p. 16); página 59, 1 (Elaboración propia a partir de difierentes fuentes. Dibujo realizado por Roberto

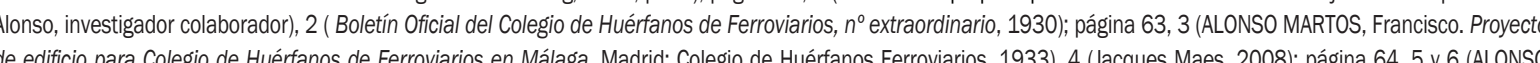

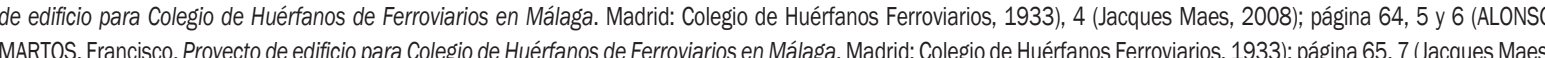

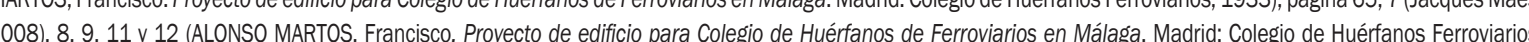
1933), 10 (Mar Loren-Méndez, 2016), 11 (ALLONSO MARTOS, Francisco. Proyecto de edificio para Colegio de Huếranos de Ferroviarios en Málaga. Madrid: Colegio de

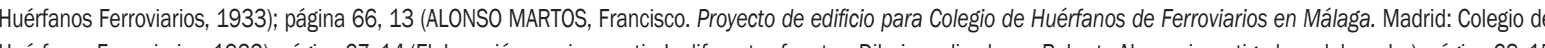

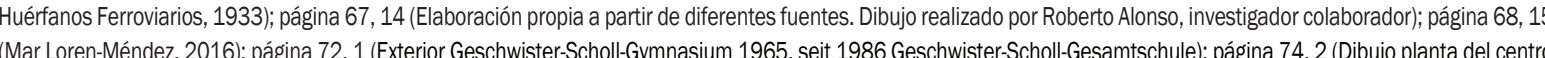

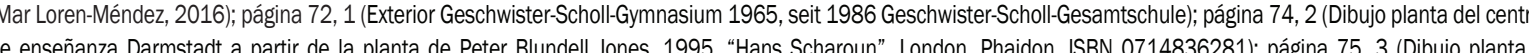
xonometría de aula para el grupo inferior, grupo intermedio y el grupo superior para el provecto del colegio en Darmstadt a partir de hitp hlescolano.blosspotcon

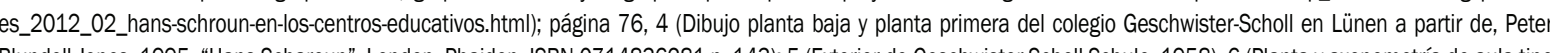
Blundell Jones, 1995, "Hans Scharoun", London, Pharidon. ISBN 0714836281.p. 143); 5 (Exterior de Geschwister-Scholl.Schule, 1958), G 6 Plantay axonometría de aula tipo en el colegio Geschwister-Scholl en Lünen a partir de, Peter Blundell Jones, 1995, "Hans Scharoun", London, Phaidon. ISBN 0714836281 p. 142), 7 Vista interior aula

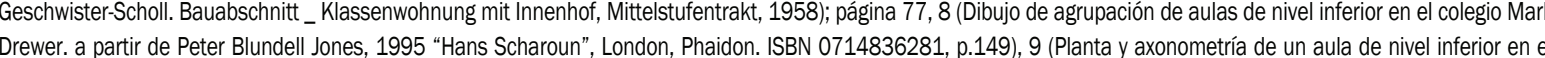
colegio Marl-Drewer. a partir de Peter Blundell Jones, 1995 "Hans Scharoun", London, Phaidon. ISBN 0714836281, p.149); página 78, 10 (Teatro del colegio Geschwister.

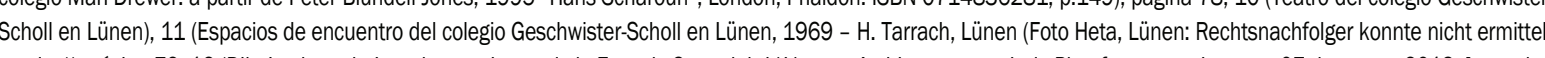

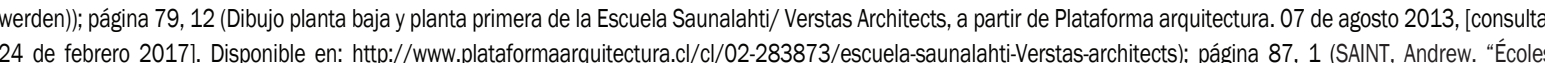

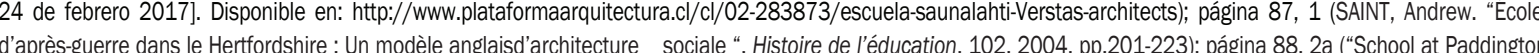

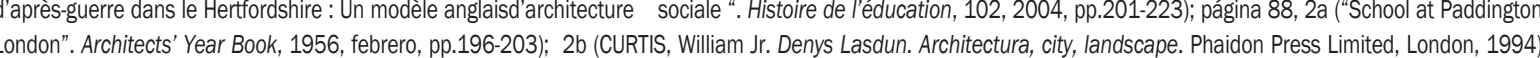

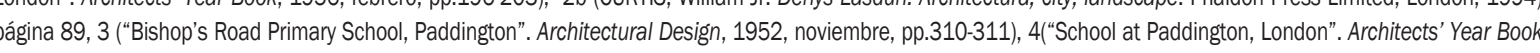
1956, febrero, pp.196-203), 5a "'School at Paddington, London". Architects's Year Book. 1956, feberero, p.196-203), 5b ""Bishop's Road Primary School, Paddington"

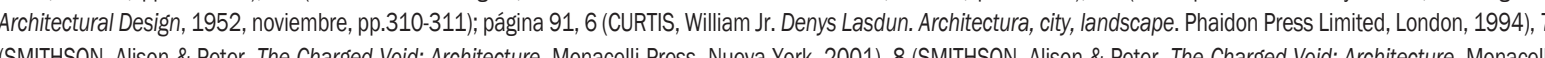

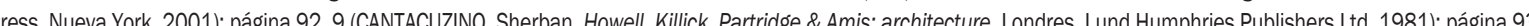

10 (CANTACUZINO, Sherban. Howell, Killick, Partridge \& Amis: architecture. Londres, Lund Humphries Publishers Ltd, 1981), 11 "PPimlico Comprehensive". Architectura

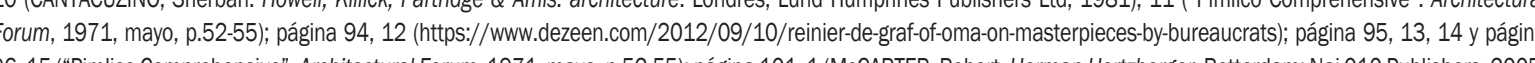
96, 15 ("Pimlico Comprenensive". Architectural Forum, 1971, mayo, p.52-55); página 101, 1 (McCARTER, Robert. Herman Hertzerger. Rotterdam: Nai 10 Publishers, 2005 ,

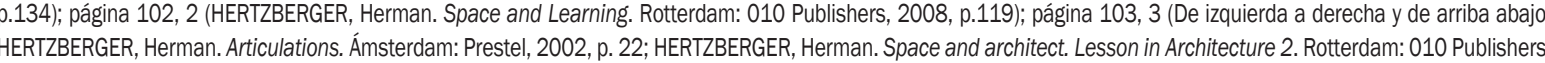

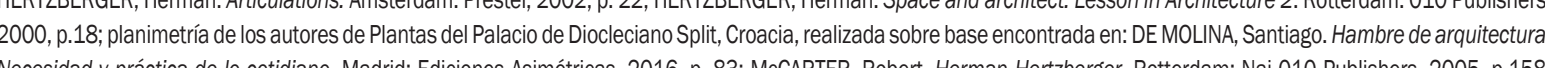
政

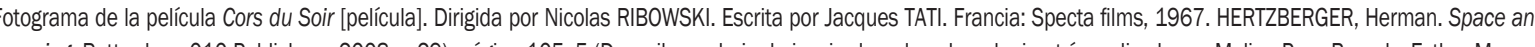

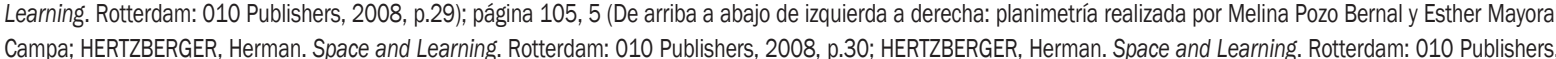

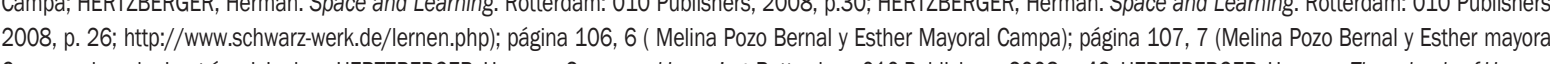

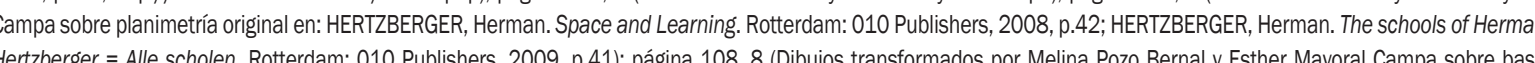
original en: HERTZZERGER, Herman. Space and Learming. Rotterdam: 010 Publishers, 2008, p. 83);página 109, 9 (Melina Pozo Bernal y Esther Mayoral Campa sobre planimetría existente en: MCCARTER, Robert. Herman Hertzberger. Rotterdam: Nai 1010 Publishers, 2005, p. 144 Y HERTZZERGGER, Herman. The schools of Herman Hertzberger

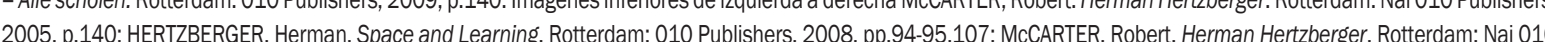

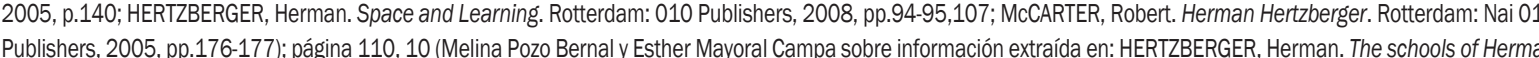
Hertzberger = Alle scholen. Rotterdam: 010 Publishers, 2009, pp.130,118. Fotograffias de arriba a abajo McCARTER, Robert. Herman Hertzberger. Rotterdam: Nai 010 Publishers, 2005, pp.184, 188, 185); página 111, 11 (Melina Pozo Bernaly Esther Mayoral Campa sobre información extraída en: McCARTER, Robert. Herman Hertzberger. Roterdam. Warto

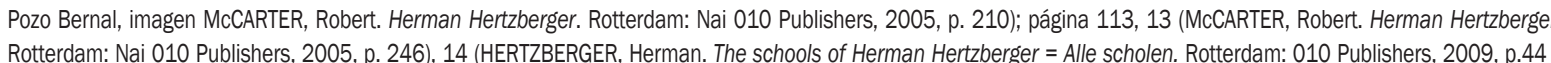
Mextraída en:

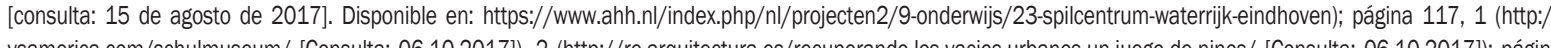

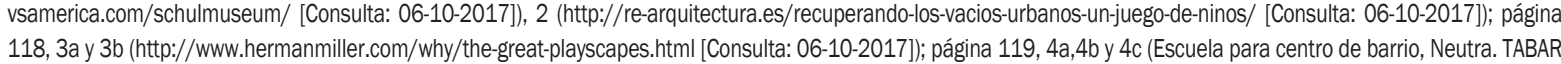

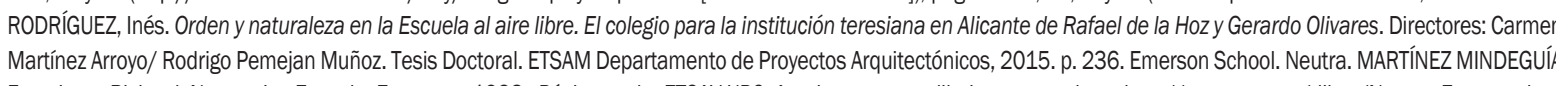

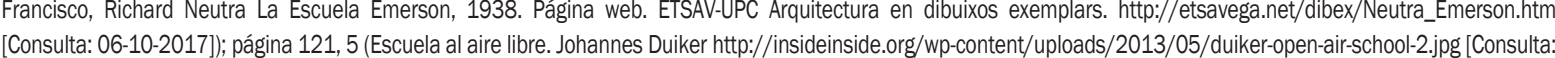

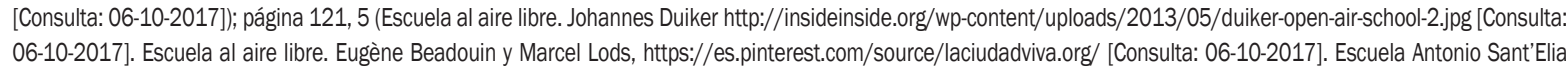

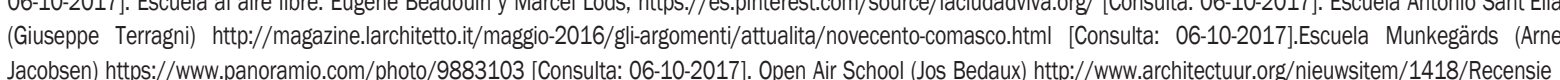

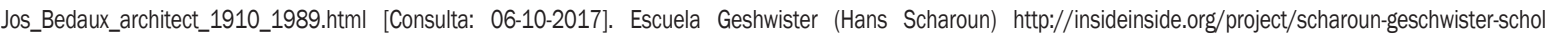

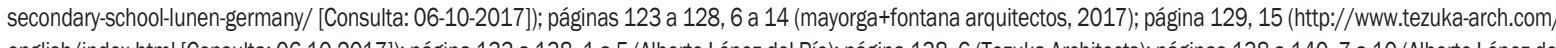

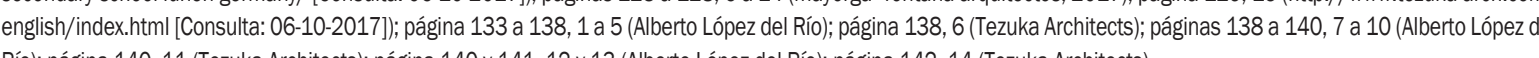

